\title{
New perspectives on binding in visual working memory
}

\author{
Sebastian Schneegans* \& Paul Bays
}

\begin{abstract}
How does visual working memory store the binding between different features of a visual object (like colour, orientation, and location), and does memorizing these bindings require additional resources beyond memorizing individual features? These questions have traditionally been addressed by comparing performance across different types of change detection task. More recently, experimental tasks such as analogue (cued) recall, combined with analysis methods including Bayesian hypothesis testing and formal model comparison, have shed new light on the properties of working memory. A significant new perspective is that noise in neural representation limits the precision of recall, and several recent models incorporate this view to account for failures of binding in working memory. We review the literature on feature binding with a focus on these new developments, and discuss their implications for the interpretation of classical findings.
\end{abstract}

\section{Introduction}

The objects and scenes that we perceive in the world are composed of a multitude of different visual features: colour and texture, shape and size, orientation and movement. To faithfully memorize such scenes, we need to form an internal representation not only of these individual features, but also of their specific conjunctions that characterize individual objects - such as a small round red ball, or a large brown box with rectangular sides.

It is a long-standing view that assessing, comparing or memorizing conjunctions of features poses a particular challenge beyond performing the same operations for individual features (Treisman, 1996). This view was initially inspired by observations in visual search tasks, where a distinction was claimed between fast, parallel search for targets characterized by a single feature, and slow sequential search for targets defined by a conjunction of features (though subsequent research challenged this simple dichotomy; see Wolfe, 1998). Based on such findings, Treisman and colleagues formulated Feature Integration Theory (Treisman and Gelade, 1980; Treisman, 1988): features belonging to different feature dimensions (by which we mean the spaces of all possible feature values of a certain type, such as colour) can be processed in parallel, for example in the form of feature maps over space. But assessing the conjunctions between features requires focused attention, which binds the features at a single location together into a temporary object representation. To account for priming effects and trans-saccadic memory, the theory was extended by proposing that a limited number of such 
temporary object representations could be formed and maintained in memory as "object files" (Kahneman et al., 1992; Irwin and Andrews, 1996).

This idea of an object file as the unit for storing features in a bound form has proved a highly influential conceptualization and has inspired many subsequent studies. It must be recognized, however, that it is primarily a metaphor with limited explanatory value in itself: It does not specify any concrete mechanism of binding features together, and in particular no neural implementation. Neural systems are not organized into files that can be filled with arbitrary content, but are formed of populations of neurons whose response to specific visual features is determined by their connectivity within the larger network.

In this paper, we will review behavioural studies that investigate the properties and limitations of feature binding in visual working memory (VWM), with a focus on newer approaches that recognize that encoding, maintenance and recall in working memory are noisy processes with limited precision. We will then describe several recent models of working memory that address the issue of feature binding and propose concrete mechanisms for implementing representations of feature conjunctions in a neural system. We will discuss implications of the proposed binding mechanisms for the interpretation of various behavioural results, and critically assess the view that memorizing feature binding requires additional resources that are not engaged in memory for individual features. Finally, we will review evidence for specific impairments of feature binding in ageing and different clinical conditions.

\section{Objects and features}

Much research related to binding in working memory (WM) has been framed in terms of whether the units of WM - which determine capacity limits - are individual features or inherently bound objects. This question was already hinted at in earlier work (Irwin and Andrews, 1996), but it gained significant traction with the influential paper of Luck and Vogel (1997; extended in Vogel et al., 2001). Luck and Vogel observed that the ability to detect a change in a stimulus array of a given set size (i.e. number of objects) was equal whether the objects varied in a single feature (e.g., coloured squares) or in up to four features (bars differing in colour, orientation, size, and presence or absence of a gap; Figure 1A). They also reported identical performance with single-colour objects and objects composed of two colours. The authors concluded that the units of working memory are integrated object representations rather than individual features. This "strong object hypothesis" goes beyond the Object File Theory, which assumed that memory for unbound features coexists with a limited number of bound representations.

Several subsequent studies attempted and failed to replicate Luck and Vogel's finding regarding two-colour objects (Wheeler and Treisman, 2002; Olson and Jiang, 2002; Xu, 2002b; Delvenne and Bruyer, 2004; Parra et al., 2011a). These studies generally found that combining two colours into a single object provided no benefit over remembering the same number of colours in separate objects, consistent with capacity limited by number of features, not objects. $\mathrm{Xu}(2002 \mathrm{~b})$ found analogous results for objects combining two orientations.

One more recent study again argued in favour of the strong object hypothesis (Luria and 

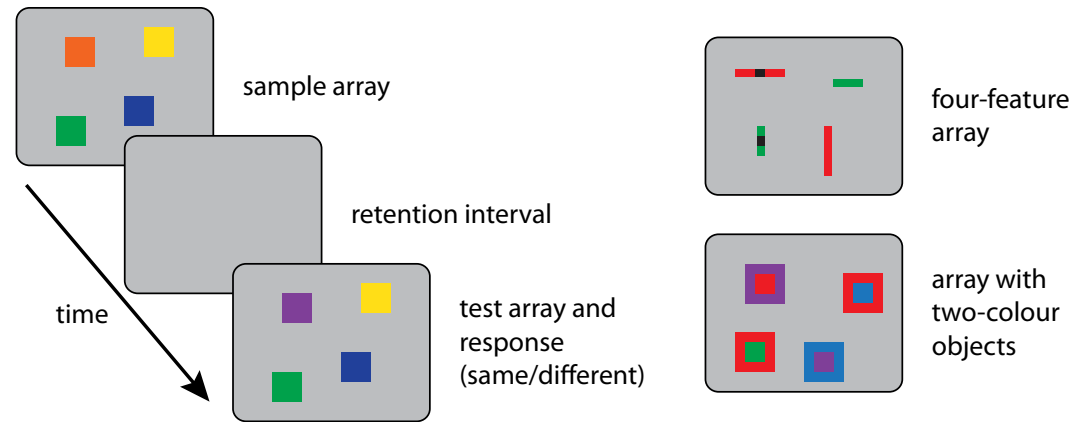

B Wheeler \& Treisman, 2002

C Treisman \& Zhang, 2006
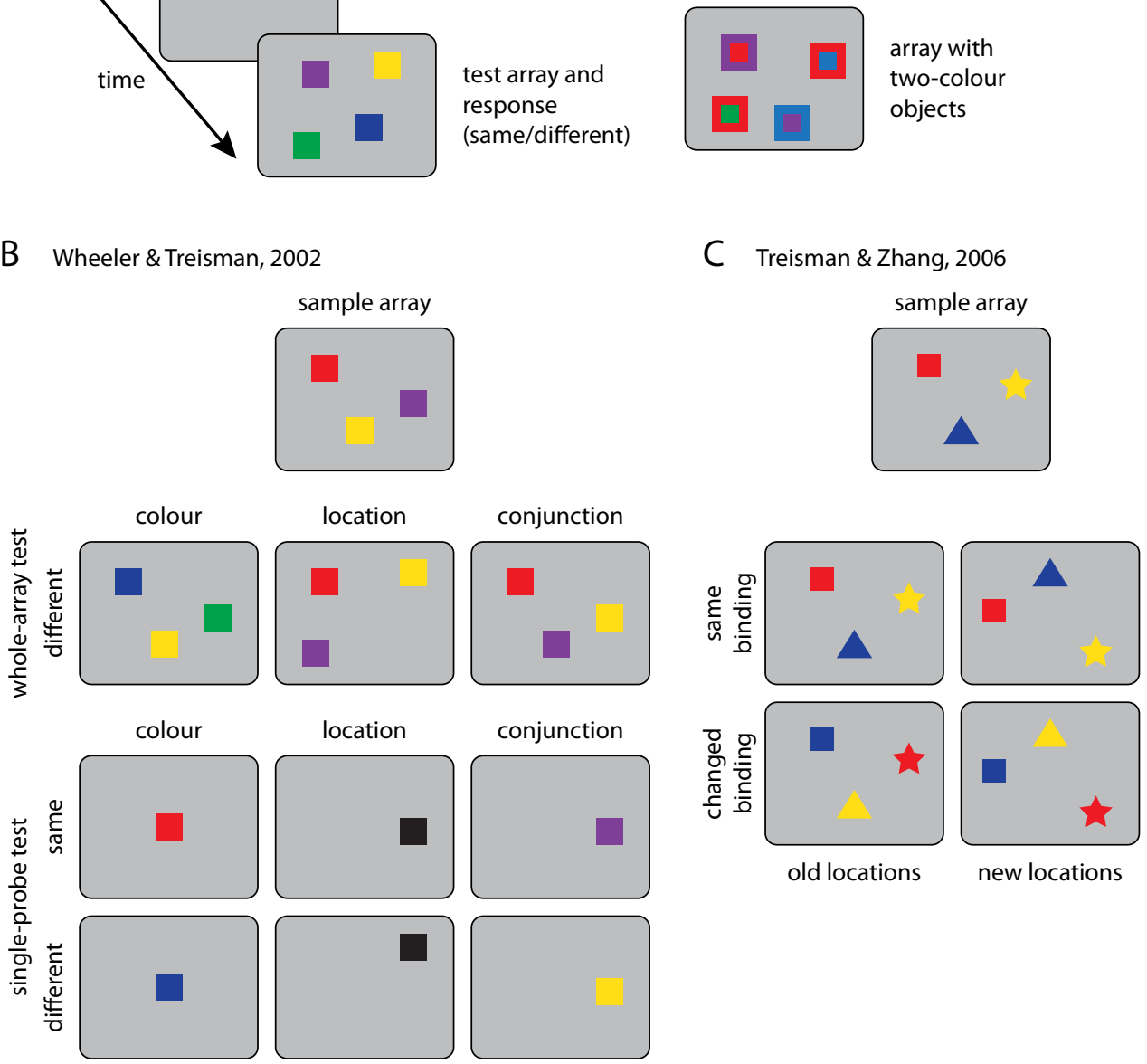

Figure 1: Change detection tasks to test working memory performance for features and conjunctions. (A) Task structure and stimulus arrays used by Luck and Vogel (1997). Participants briefly view a sample array, followed by a retention interval and then a test array, and have to report whether the two arrays are the same or different (here shown for a "different" trial in a colour change detection task). On the right, example stimulus arrays are shown with four features per item (orientation, colour, size, and presence of a gap) and for objects comprising two colours each. (B) Examples of sample and test arrays used in Experiment 3 of Wheeler and Treisman (2002). Possible test arrays are shown realizing changes in colour, location, or colour-location conjunctions compared to the given sample array, using either whole-array or single-probe tests. For the whole-array test, the arrays in "same" trials are always identical to the sample array. For feature changes (colour or location), the features of two items are changed to new values (to match the number of items affected by a single conjunction change). In the single-probe tests, colour memory is tested with centrally presented colours, and location memory with black squares, while conjunction memory is tested with a coloured square at the location of one item in the sample array. (C) Examples of test and sample arrays from Experiment 1 of Treisman and Zhang (2006). For this experiment, only changes to novel features had to be detected (so all test arrays shown here represent "same" trials for the given sample array), but task-irrelevant changes in locations or bindings of features could be introduced at test. 
Vogel, 2011), this time based on EEG results showing no difference in delay activity between colour-conjunction objects and the same number of single-colour objects during the later phase of maintenance (but see Wilson et al., 2012). While this study found a performance cost for dual-colour compared to single-colour stimuli, the authors argued that this was merely due to comparison errors for the visually more complex conjunction stimuli (Awh et al., 2007). It is not clear, however, why the same effect of higher complexity on comparison errors would not be observed for items that combine different feature dimensions. Moreover, Taylor et al. (2017) found that effects of item complexity are significantly better explained by reduction in memory capacity than an increase in comparison errors. Overall, the strong object hypothesis is poorly supported by current evidence.

\section{Support for independent feature stores}

Luck and Vogel (1997) had employed the experiment with dual-colour stimuli to exclude an alternative to the strong object hypothesis, namely the idea of independent working memory stores for different feature dimensions. This view, which was espoused among others by Magnussen and Greenlee (1999) and Wheeler and Treisman (2002), assumes that there is a separate working memory capacity for each feature dimension, such that features can be added in one dimension without incurring any cost on memory performance for other, qualitatively different feature dimensions. While it is now widely accepted that there is a substantial benefit to storing combinations of different features (e.g. two colours and two orientations) instead of more of the same feature (e.g. four orientations), the question of whether there is a cost of increasing the number of feature dimensions is still actively investigated.

The original findings of Luck and Vogel (1997) indicating equal performance for singlefeature and multi-feature objects were reproduced by several subsequent change detection studies (Olson and Jiang, 2002; Delvenne and Bruyer, 2004; Riggs et al., 2011; and one experiment of Wheeler and Treisman, 2002, although other experiments did show a small cost for additional features). In a recent study, Wang et al. (2017) employed a modification of the typical change detection design in which they varied the number of different feature values in each dimension (by allowing groups of items to share e.g. the same colour), rather than the number of relevant feature dimensions. They found that having more different feature values in one dimension reduced performance for detecting changes within that dimension, but had no significant effect on detecting changes in other dimensions. This is consistent with the concept of independent feature stores.

One weakness of these earlier studies is that their conclusions were based on statistical null effects (no significant difference in performance for added feature dimensions). In contrast, the recent study of Shin and $\mathrm{Ma}$ (2017) addressed several questions regarding the storage of different features using formal model comparisons. While earlier work often treated memorization as a binary process (a feature is either memorized or not, and if it is, then a change in that feature will be detected), this study was based on more recent conceptualizations of working memory: It assumed that the precision of memorized features is limited due to random noise (Bays and Husain, 2008; van den Berg et al., 2012; Ma et al., 2014), and that forming a decision (change or no change) based on noisy representations of sample and test stimuli is a 


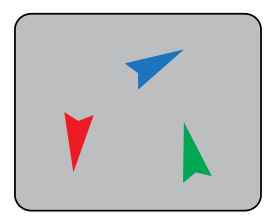

3 objects,

6 features

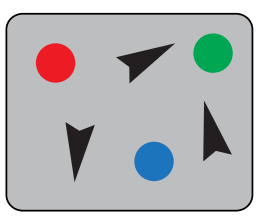

6 objects, 6 features

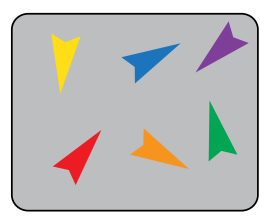

6 objects,

12 features

Figure 2: Possible memory sample arrays to test how memory performance is affected by number of objects and number of features to be memorized. These sample arrays may be used either for a change detection or an analogue recall task. The left display is matched to the middle display with respect to the number of relevant features to be memorized (three colours and three orientations), the right display is matched to the middle one in the number of objects in the array.

process of probabilistic inference.

Shin and Ma obtained behavioural data for a change localization task with varying change magnitude, and produced quantitative model fits based on different assumptions about feature storage. Among other results, they found that a model with independent memory resources for colour and orientation accounted for the data significantly better than one with fully shared capacity. One important aspect taken into account by the formal modelling was the increased decision noise when comparing multiple features: the simple fact that more individual comparisons are performed when detecting or localizing a change in an array with more features can lead to a higher error rate in multi-feature arrays, even when memory resources for different feature dimensions are entirely independent.

\section{Object benefit and effects of feature separation}

While these studies support largely separate feature stores, there is also substantial evidence that their independence is incomplete. One relatively uncontroversial finding is that the number of objects still matters, with better performance when features are combined into fewer objects (the "object benefit"; this is sometimes referred to as the "weak object hypothesis"). Specifically, change detection performance for arrays of $N$ conjunction stimuli (e.g., 3 coloured oriented arrows; Figure 2) tends to be significantly better than for a mixed array with $N$ items for each feature dimension (e.g., 3 black arrows and 3 coloured disks; Olson and Jiang, 2002; Delvenne and Bruyer, 2004; Wilson et al., 2012). Fougnie et al. (2013) also found similar results in an analogue recall task (Figure 3). The study of Shin and Ma (2017) likewise supports an object benefit based on formal model comparisons, attributing it to "leaking" of memory resources for each feature dimension to the stimuli for which that feature is not relevant (or non-existing). Nonetheless, the performance for mixed displays in these studies is still better than performance for $2 N$ conjunctions (e.g., 6 coloured arrows; Marshall and Bays, 2013, but see $\mathrm{Xu}, 2002 \mathrm{a}$ for partially contradictory findings).

It has been observed in various studies that the object benefit (or, conversely, the cost of memorizing additional features of a stimulus) depends on the distance and connectedness of the individual features within the object. For instance, Xu (2002a) found that the cost of mon- 


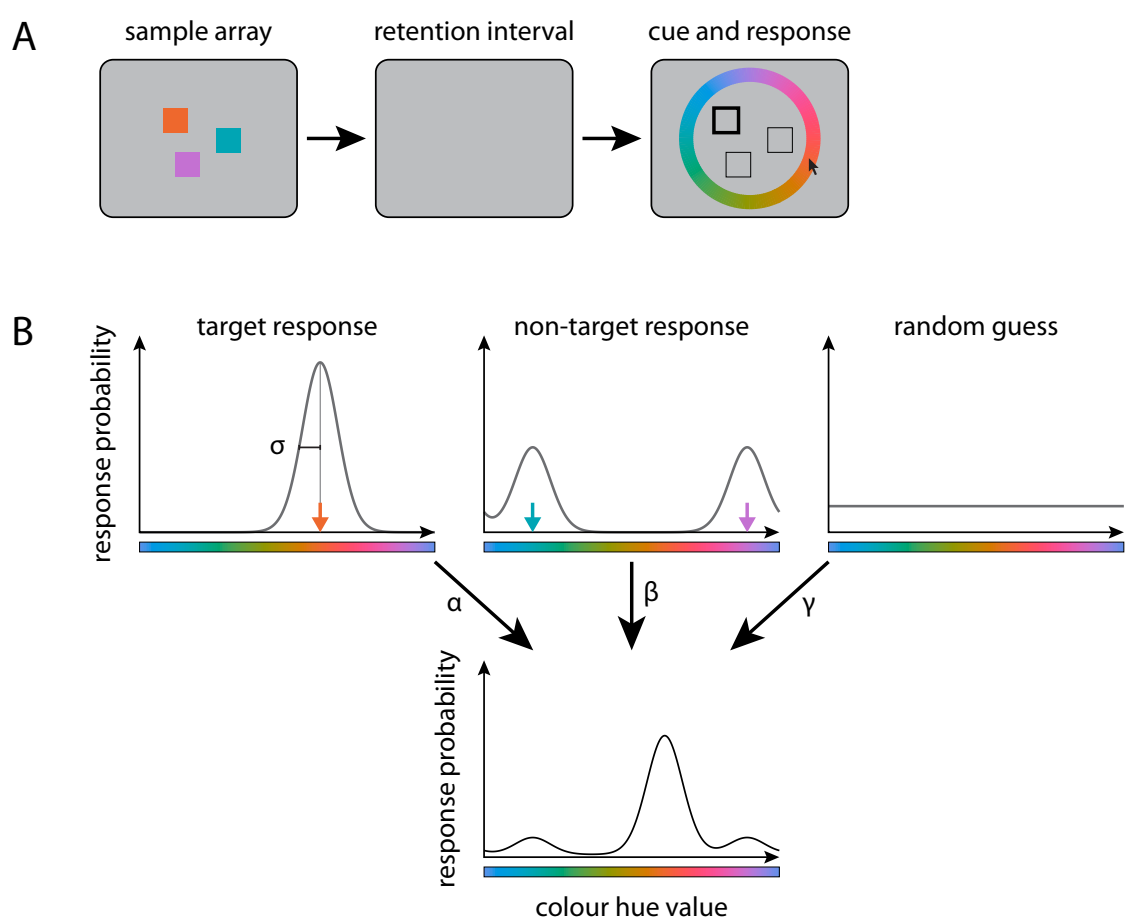

Figure 3: An analogue recall (or continuous reproduction) task. (A) Participants view a sample array, followed by a retention interval, and then a test display in which they have to report a feature value of one item on a continuous scale. In the example here, participants are cued with location and have to report the colour of the item at that location on a colour wheel. (B) Response distributions are often analysed using a mixture model (here the three-component model of Bays et al., 2009). The model assumes that responses either reflect the feature value of the cued item (the target) with some variability $\sigma$, a feature of one of the other (non-target) items in the sample array with the same variability, or are the result of a random guess. A maximum likelihood fit of the model to empirical data yields estimates of the variability measure $\sigma$ and the weights of the different mixture components $\alpha, \beta$, and $\gamma$ (with $\alpha+\beta+\gamma=1$ ). 
itoring two features compared to one feature for changes in an object array decreased when the different features were combined into the same part of an object (e.g. a coloured oriented bar compared to a black bar over a coloured circle). The same dual-feature monitoring cost is lower when object parts (here, coloured "cap" and oriented "stem" of mushroom stimuli) are presented as conjoined objects, rather than spatially separated (Xu, 2002b; but see Delvenne and Bruyer, 2006, for limitations of this finding, and Lee and Chun, 2001, for contrary results). Xu (2006) further showed specific effects of feature distance and connectedness in such mushroom stimuli by systematically manipulating the degree of separation between the stem and cap components.

Wang et al. (2016) further explored the object benefit by varying the presentation type simultaneous or sequential presentation, either at the same or different locations - in addition to the conjunction state of items (joined or separated, using either mushroom stimuli or colourorientation combinations). They found that the object benefit critically depended on whether object location was relevant and informative in the task, and concluded that it is in fact foremost a shared-location benefit, rather than a benefit of shared objecthood (see also Davis and Holmes, 2005). While this is consistent with most previous findings on the object benefit, one result is potentially at odds with it: extending earlier results from Delvenne and Bruyer (2004), Ecker et al. (2013) found that the cost of dual-feature monitoring for shape-colour combinations is reduced when they appear to be part of the same object rather than object and background, even if the difference in visual appearance (and spatial separation) that generates that effect is minimal.

\section{Further limits on feature independence}

Beyond the performance costs that arise when features are spatially separated, several more recent studies have also found a cost of adding more feature dimensions even for strongly integrated objects (although this cost is still lower than that of adding more items in the same feature dimension). Fougnie et al. (2010) tested independence of feature capacity in an analogue recall task. Subjects had to memorize arrays of coloured, oriented triangles, and after

a delay report a feature of one item cued by its location. In separate blocks, subjects were tested only on colour, only on orientation, or on either feature. While the proportion of responses reflecting the target feature (rather than a non-target or a random guess; see Figure 3B) was unaffected by condition, recall precision was found to be significantly reduced in the either-feature blocks. The authors argued that the failure to detect a cost of additional feature dimensions in previous change detection tasks was due to a lack of sensitivity when using only large categorical changes, and indeed found a dual-feature cost in a subsequent change detection task specifically when using small changes in feature values. A decrease in reproduction precision was also observed when memorizing two features instead of only one feature of a single memory item (Swan et al., 2016; see also Palmer et al., 2015).

Oberauer and Eichenberger (2013) employed a classic change detection task, but increased the number of feature dimensions up to six. Using Bayesian statistics, they found effects of both set size and number of features in the display on change detection performance, largely independent of the number of possible values in each dimension and the available en- 
coding time. Unlike Fougnie et al. (2010), they also observed the same effects regardless of change magnitude. In contrast to Luck and Vogel (1997) and other studies, Cowan et al. (2013) found a significant (although modest) cost of monitoring for changes in two features at the same time even for highly integrated stimuli (coloured shapes), and even when the feature that could have changed was indicated at test (to control for decision noise, as discussed above). Hardman and Cowan (2015) directly attempted to replicate the multi-feature experiment of Luck and Vogel (1997) (also controlling for decision noise). Using Bayesian statistics, they found strong evidence for an effect of the number of relevant feature dimensions on change detection performance, in addition to the set size effect observed in the original study. Nonetheless, the study still found support for partial independence of feature stores, in that the estimated total number of memorized feature values in the multi-feature condition was higher than in the easiest single-feature condition.

\section{Independence of recall for features of single objects}

A different question regarding the organization of working memory is whether different features of a single object are memorized and recalled as a unit (i.e. if one feature of an object can be recalled, then all of its other features should be recalled as well) or whether they are memorized and recalled independently of each other (such that e.g. the colours of one subset and the orientation of another subset of sample items can be reported). While object-based approaches inherently favour the former hypothesis, the concept of separate capacity limits is actually compatible with both possibilities. The question can be addressed directly in analogue recall tasks if participants have to report multiple features of a cued item. Using this approach, Fougnie and Alvarez (2011) and Fougnie et al. (2013) found that memory failures, operationalized as metrically large recall errors, occurred largely independently for reporting an item's colour and orientation (but were correlated for closely related features, namely width and height of a rectangle). Bays et al. (2011) directly fit the behavioural data in such a task with an extended mixture model that distinguished independent and correlated swap or guess errors, and the best fit was close to the prediction of fully independent feature recall. One earlier study had found nearly opposite results, with strong correlations between the memorized items for different feature dimensions (Gajewski and Brockmole, 2006). The authors of the subsequent studies argued that this was likely an artefact resulting from a very short presentation time and widely spaced objects, leading subjects to focus their spatial attention on only a subset of items to achieve at least partial memorization of the sample array. Such an effect of shorter presentation time was subsequently confirmed by Peich et al. (2013).

There is also evidence for at least partial independence in the encoding of an object's features into WM. By using varying, brief presentation times of a sample array, followed by a mask, Woodman and Vogel (2008) showed that colours can be encoded more quickly than orientations, but that their encoding is slowed down when both features have to be memorized. Moreover, the authors found a lower amplitude of the contralateral delay activity (CDA), an EEG measure related to working memory load, for conditions where only colour was relevant compared to orientation or dual-feature conditions. Memory for different features can be differentially affected by retro-cues indicating which feature dimension is going to be tested (Park 
et al., 2017), supporting some independence in storing different features of the same items, but also a trade-off of capacity between them. A number of studies have also investigated to what degree task-irrelevant features are memorized, finding low-precision, but above-chance performance in surprise tests (Swan et al., 2016; Shin and Ma, 2016; see also Shin and Ma, 2017), some degree of interference from irrelevant feature changes (Gao et al., 2011; Shen et al., 2013; Hyun et al., 2009), and limitations on the ability to ignore features of specific items in mixed displays (Vidal et al., 2005; Marshall and Bays, 2013).

It has also been observed that the feature conjunctions in a sample array affect the comparison to a single test item, even when only the individual features are task-relevant (Quinlan and Cohen, 2011; Vergauwe and Cowan, 2015). This has been interpreted as evidence for object-based working memory representations, but could also be explained by features separately encoded in different stores being bound together again at recall (e.g. through spatial attention directed to the same location in multiple feature maps, as proposed in section "Space as a binding dimension", below). These results do indicate, however, that feature bindings are stored at least in an implicit form even when they are not task-relevant (but see Sala and Courtney, 2009 for an opposing view).

\section{Interim summary}

Based on the results reviewed here, the strong object hypothesis can be considered largely refuted, both with respect to capacity limits and independence of recall for different features of individual objects. There is strong support for at least a partial independence of memory capacities for different feature dimensions, but also substantial evidence that there is some cost associated with adding more feature dimensions. The somewhat discrepant results regarding the magnitude of this cost may in part stem from the specific choice of feature dimensions used in the sample arrays, with some supposedly distinct feature dimensions still tapping into the same feature stores (e.g. various spatial features in Oberauer and Eichenberger, 2013). Despite the overall support for independence of memory for different feature dimensions, there is also robust evidence that combining features into fewer individual objects conveys a benefit for working memory performance. This benefit may be mediated by the number of locations over which the features are distributed, suggesting an important role of space that is consistent with models of working memory based on multiple interconnected feature maps (Treisman, 1988; see also Franconeri et al., 2013).

\section{Binding features in VWM}

\section{Evidence for a binding deficit}

While the considerations about features and objects in the previous section provide important constraints for the problem of feature binding, they do not directly address the questions of how and to what degree the specific conjunctions between different features of an object are memorized. Several classical studies suggested that WM for conjunctions was less reliable than memory for individual features (Stefurak and Boynton, 1986; Treisman et al., 1977). We will 
refer to a performance decrement between a task requiring memorization of feature bindings and one that requires only memory for individual features as a binding deficit in this review.

The issue was further investigated in the influential study of Wheeler and Treisman (2002), who employed comparisons between different conditions in a change detection task to assess memory performance for feature conjunctions in comparison to single features. For instance, for a sample array of coloured squares, they instructed participants in separate blocks to monitor for changes either in colour, location, both features (independent of binding), or the specific conjunctions of colours and locations. Only the relevant features were ever changed at test (to new values in the single feature and both-feature condition, by recombination in the conjunction condition), and they employed either a whole-array or a single-probe test (Figure 1B).

The authors found that change detection performance was specifically impaired in the binding condition compared to the both-features condition, but only when using the whole-array test, and not for single-probe tests. This pattern was reproduced when testing binding of colours to shapes (with location made uninformative by randomly scrambling items at test). Wheeler and Treisman concluded that, in addition to separate feature stores with their own capacity, there is an additional capacity-limited storage for memorizing feature bindings. They further proposed that this latter storage is dependent on sustained attention, making it susceptible to interference from the presentation of multiple stimuli in the whole-array test (however, several subsequent studies have failed to replicate the difference between whole-array and single-probe conditions; see section "Problems of assessing the binding deficit", below).

A specific limitation in binding memory was also reported in an experiment requiring the continuous monitoring of a rotating, cyclically occluded array of coloured disks (Saiki, 2003a). Subjects showed a significantly decreased performance for detecting colour swaps compared to introduction of new colours, although subsequent research indicated that this effect may be specifically caused by the movement of the array (Saiki, 2003b).

\section{Role of attention}

Various studies have tried to further elucidate the deficit in change detection performance for feature binding, and in particular the role of attention in binding memory (sometimes framed in terms of the involvement of the episodic buffer and central executive within the influential model of Baddeley and Hitch, 1974, and Baddeley, 2000). One key method employed is the dualtask paradigm, in which a second, attention-demanding task is performed during the retention interval of a working memory task that tests either single features or conjunctions. Using this approach, Allen et al. (2006) found that a concurrent backwards counting task or a verbal memory task impaired overall change detection performance, but did not have a selective impact on memory for feature bindings. These results were confirmed and extended in later studies with various concurrent tasks (Allen et al., 2012; Morey and Bieler, 2013; Vergauwe et al., 2014; see also Yeh et al., 2005; Delvenne et al., 2010), and were found to hold even when features were presented separately and had to be bound internally (Allen et al., 2009; Karlsen et al., 2010; but see Gao et al., 2017). These results indicate that binding memory does not make specific demands on sustained attention (but see Brown and Brockmole, 2010 for a conflicting finding). 
However, certain task conditions have been found to specifically impact binding memory performance. Allen et al. (2006) observed that memory for feature binding declined more than memory for individual features when the items of the sample array were presented sequentially rather than simultaneously (confirmed by Brown and Brockmole, 2010; Allen et al., 2014). A specific susceptibility of feature bindings to interference from subsequent distractor stimuli has also been observed (Ueno et al., 2011; Allen et al., 2015), but only if these distractors were similar to the sample items. And while most attention-demanding concurrent tasks do not cause a selective binding deficit, such a deficit has been observed for concurrent tasks that strongly tax visuo-spatial or object-based attention, including demanding multi-object tracking (Fougnie and Marois, 2009), mental rotation (Shen et al., 2015) or visual search (Zokaei et al., 2014; but see Johnson et al., 2008 for an earlier study that failed to find a selective effect of visual search on feature binding).

These findings suggest that there is no specific reliance of feature binding on the kind of attentional resources conceptualized in the central executive, but there is evidence for a specific susceptibility to interference from subsequent visual stimulation and challenging tasks that require visuo-spatial attention.

\section{Problems of assessing the binding deficit}

While the studies reviewed above yield a relatively consistent picture regarding the role of attention, their results are less clear regarding the more elementary question of whether there is a binding deficit in WM in the absence of concurrent tasks, i.e. a specific limitation for memorizing the binding between features. Unlike Wheeler and Treisman (2002), some studies found evidence for a binding deficit even when using a single-probe test (Brown and Brockmole, 2010; Ueno et al., 2011). In contrast, Johnson et al. (2008) tried to directly replicate the findings of Wheeler and Treisman, and they observed that performance for binding changes was not different from performance for changes in the more difficult of the individual features. This result did not differ between single-probe and whole-array tests.

Some of the discrepancy between studies can be attributed to the fact that there is no clear consensus about what the criterion for a binding deficit should be in a change detection task. Since an incorrect response provides little information about the way in which encoding, maintenance or comparison failed in a single trial, binding deficits can only be detected by comparing accuracy measures in different task conditions that are putatively matched in their memory demands except for the role of feature binding. To achieve this for whole-array tests, Wheeler and Treisman (2002) compared a single feature swap in the binding condition with the replacement of two feature values in the single-feature and either-feature conditions, such that the number of items affected by the change is matched. Allen et al. (2006) argued that when using single-probe tests in a binding-change condition, the sample array should also contain feature repetitions in some trials (which they excluded in the analysis) so that participants could not remember only a subset of items and rely on exclusion principles to determine the correct response (e.g., if an item matches one feature of a remembered item but not the other, it must be a recombination).

It is also disputed whether the binding condition should be compared to a condition requir- 
ing the simultaneous monitoring of two features (independent of their binding), or to the more difficult of the two single-feature conditions. In cases where there is a significant difference in performance for the two feature dimensions involved, the either-feature condition might yield better performance because some changes are in the easy-to-detect feature, while the binding condition necessarily requires memorizing the more difficult feature as well, a point made by Johnson et al. (2008). These authors also noted the possibility that summary statistics of features in an array (e.g. the average colour; see Brady et al., 2011 for a review) could be used in change detection; the summary statistics are affected by introducing novel feature values, but not by recombination of features. Additional factors that may affect performance are whether the stimuli in the single-feature conditions vary only in that single relevant feature or in both features (e.g. black shapes vs. shapes with different, but task-irrelevant colours; Allen et al., 2012), and whether different conditions are mixed or blocked (Morey and Bieler, 2013). It has also been suggested that performance in a change detection task, in particular with wholearray test, generally underestimates memory capacity for feature conjunctions compared to cued recall tasks, due to automatic overwriting or rebinding of features when the test array is presented (Alvarez and Thompson, 2009).

A final issue arises from the finding that recall errors occur independently for different features of a single item, as discussed above (Bays et al., 2011; Fougnie and Alvarez, 2011). Taking this into account, Cowan et al. (2013) predicted that the proportion of correctly recalled feature conjunctions from a sample array should be the product of the proportions of correctly recalled features in each feature dimension. They argued for a capacity limit with respect to the number of items held in WM, but assumed that for each item only a subset of features might be memorized. A quantitative model based on these assumptions (and without any additional capacity limit for bindings) accounted well for the experimentally observed performance in a change detection task with single-feature and binding changes. The authors proposed that a small remaining deficit in binding performance might be due to a negative correlation between memory for the two features from trial to trial (consistent with Park et al., 2017).

\section{Interim summary}

The issues discussed above illustrate the difficulty of defining and assessing a specific binding deficit in change detection tasks that would indicate a separate substrate or mechanism for storing conjunctions (with its own capacity limitations or potential for errors), in addition to memory stores for individual features. The interpretation of many results necessarily depends on specific assumptions about the organization of memory stores and the processes used to reach a decision about the presence or absence of a change. In the following section, we will review several recent models that propose concrete mechanisms for feature binding, but primarily address analogue (cued) recall rather than change detection tasks. Analogue recall (Figure $3 \mathrm{~A}$ ) inherently requires binding in memory, since the report feature must be reproduced for the item matching the given cue feature (typically an item's location, but sometimes another feature such as colour, see Gorgoraptis et al., 2011). The graded response provides more information than the binary response in change detection tasks, and in particular allows detection of "swap errors" (Bays et al., 2009), in which the wrong item's feature value is reported. 
This kind of error indicates a failure to retrieve the correct memory item based on the cue. We will revisit the issue of a binding deficit in change detection tasks at a later point, after reviewing different models of feature binding in WM in the next section and discussing the explanations they provide for swap errors in cued recall tasks.

\section{Models of feature binding}

Various models have proposed concrete mechanisms and neural processes for memorizing and binding different features in visual WM, aiming to explain various results from behavioural experiments. We will review an influential classical model of binding and several more recent models. These newer models all share the idea that individual features are represented in a form of population code, such that the range of possible feature values (e.g., colour hues) is represented in the activity pattern of a population of neurons with varying preferred values. Related to this, memory stores are assumed to be characterized by a limited precision of representation, rather than a binary distinction between items that are memorized and those that are not.

\section{Binding by synchrony}

Several influential early papers (Vogel et al., 2001; Wheeler and Treisman, 2002) referred to the idea of binding through synchronization of neural firing as a possible explanation for their behavioural findings. This conceptual model (von der Malsburg, 1999; Engel and Singer, 2001) is based on oscillatory patterns in cortical neural activity, with neurons showing increased firing at regular intervals. It proposes that neurons contributing to the representation of a single object fire in synchrony with each other, while activity for separate objects occurs at different phases of an oscillatory cycle. Creating and maintaining such synchrony across different neural populations requires specific properties of the involved neurons and their connectivity, and while some aspects of this model are supported by recent neural evidence (Axmacher et al., 2010; Lundqvist et al., 2016), a functional role of synchrony in binding is still controversial (Harris and Gordon, 2015; Shadlen and Movshon, 1999).

A formal neural model implementing binding in VWM through synchronized spiking was presented by Raffone and Wolters (2001). The model seeks to explain putative capacity limits of VWM based on the principle that a limited number of separate phases of neural firing for different objects can be fit into an oscillatory cycle while still keeping those phases both stable and distinct from each other. The model therefore implements the strong object hypothesis, proposing that VWM capacity is only limited by the number of distinct items, and not the number of features within each item. To our knowledge no existing synchronisation model can reproduce the observed decline in recall precision with memory load (Bays and Husain, 2008), nor quantitatively fit behavioural data. We therefore turn our attention to more recent models that in many cases do provide such quantitative fits, based on various alternatives to binding by synchrony (O'Reilly et al., 2003). 


\section{Binding pool model}

The binding pool model of Swan and Wyble (2014) has at its core a population of neurons that are linked to various individual feature representations - colour and location, in the example shown in Figure 4A. Each neuron in this binding pool is connected to neurons that form a population code for single features in a largely random fashion (except that the connectivity respects some similarity metrics within feature dimensions). In addition, there is a set of discrete "token" nodes, which are likewise randomly connected to binding pool nodes. Visual stimuli are memorized in a sequential fashion: For every item, its perceived feature values are activated in the individual feature representations, together with a randomly selected token node. The activity of all binding pool nodes connected to these activated nodes is then elevated, and this elevated activity is sustained during the retention interval. To generate a response in a cued recall task, the feature value of the cue is first used to reactivate one of the token nodes via the active neurons in the binding pool, and then the cue feature and token together are used to reconstruct the response feature. For a colour change detection task, this procedure is performed to retrieve each item's memorized colour based on its location, and the retrieved features are compared to the colours in the test array. The concentration of activity for the recalled feature additionally provides a measure of response confidence.

The model has been shown to successfully reproduce effects of set size and change magnitude in change detection tasks, and in cued recall tasks it accounted for effects of set size and variability of sample items in cue and report dimensions. The model specifies relatively detailed neural processes for encoding and retrieval, although it requires each model neuron to perform complex operations that would likely require more than a single neuron to realize in a biological system (e.g., maintaining an arbitrary activity level for each model neuron over the retention interval, and performing multiple forms of multiplicative operation on activity state and inputs). Some additional restrictions are employed for fitting behavioural data, namely that only a random subset of 2-7 items from a sample array are encoded, and that a purely random response is produced if a token cannot be selected unambiguously during recall. The model does not explicitly implement a mechanism for storing combinations of more than two features, although the authors suggest that several independent feature pairs (e.g. colour-location and shape-location) might be linked to the same token node to encode all features of an object.

\section{Interference model}

More recently, Oberauer and Lin (2017) proposed an interference model of visual working memory. In this model, the binding between two visual features is conceptualized as a distribution of activation in a binding space (Figure 4B). This continuous, two-dimensional space is spanned by the two feature dimensions to be bound, e.g. colour and location. Each item to be memorized (as a conjunction of these two features) is represented as a two-dimensional von Mises (circular normal) distribution in this space, centred on the item's specific combination of feature values. Additionally, background activation is assumed to be present in this binding space as a result of neural noise.

To retrieve a memorized item in a cued recall task, the cue feature value is applied in 
A

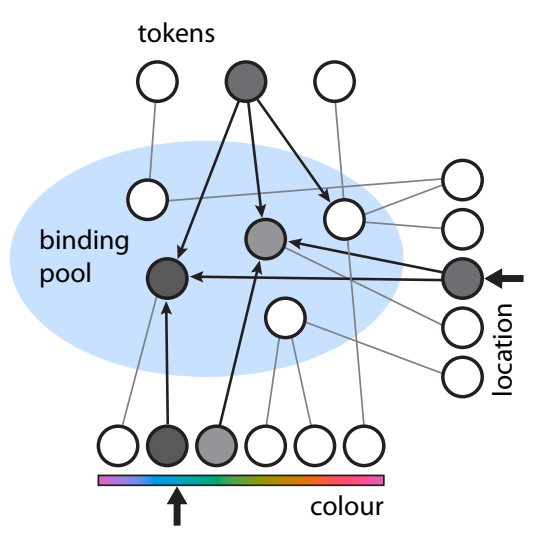

C

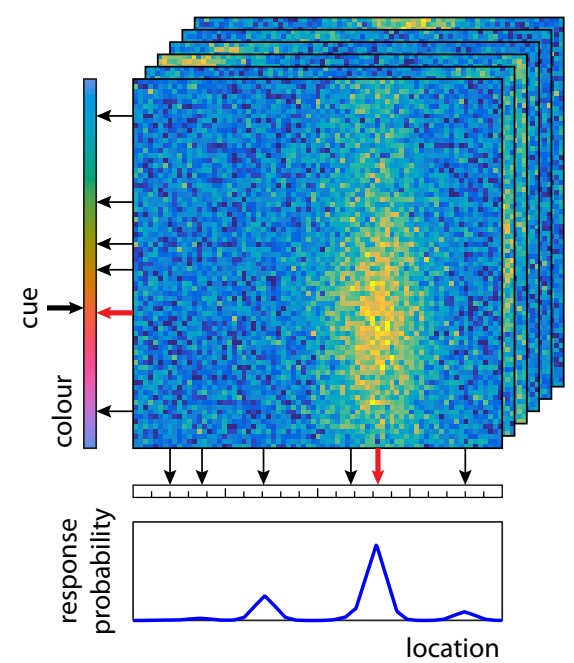

B

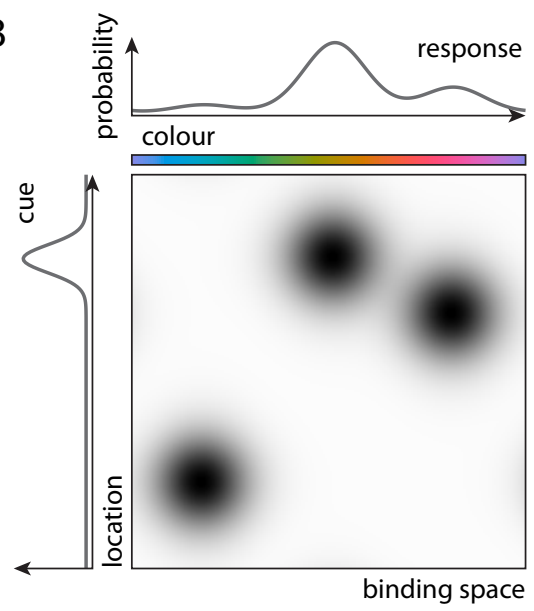

D
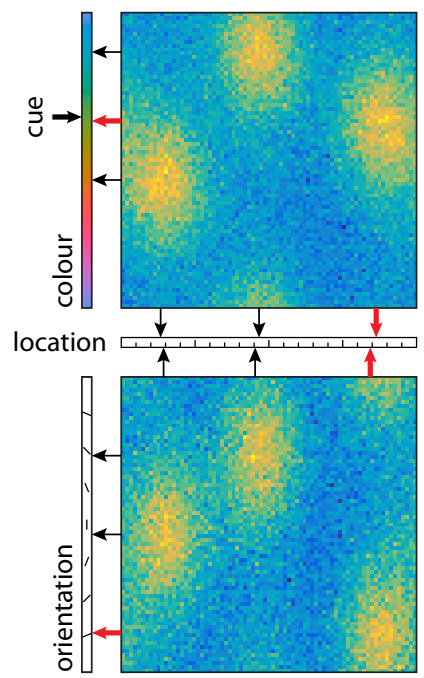

Figure 4: Neural models of feature binding in VWM. (A) Binding pool model (Swan and Wyble, 2014), during the encoding of a single item characterized by colour and location. Neurons are shown as circles, with shading indicating activation (darker meaning more active). (B) Interference model (Oberauer and Lin, 2017) during the recall of an item's colour for a given location cue. The cue is introduced as an activation distribution over one-dimensional space, and the colour-location conjunctions for a memorized sample array are reflected in the activation distribution in the binding space (indicated by shading, darker meaning higher activation). The resulting distribution of response probabilities over colour space is shown on top. Note that only one-dimensional location information is used here and in the following model, reflecting the angular location of a stimulus on a circle around fixation. (C) Conjunctive population code model (Schneegans and Bays, 2017), shown for an analogue recall task with colour cue and location response. Activity in neural population representations of multiple items is shown colour coded. For recall, the feature values of each memorized item are decoded by maximum likelihood estimation (black arrows), and the item whose colour value is closest to the given cue is selected and its decoded location value produced as a response (red arrows). The distribution of response probabilities (reflecting recall variability and swap errors) is shown at the bottom. (D) Extended model of Schneegans and Bays (2017) with spatial binding of colour and orientation. Population representations of multiple items are shown superimposed here. To report the orientation of an item given a colour cue, the model first estimates the location of the cued item, and then uses the location as a secondary cue to produce the orientation response. 
the form of a one-dimensional von Mises distribution in the corresponding feature dimension (again with the addition of a uniform component reflecting neural noise), and the multiplication of this distribution with the binding space distribution, projected onto the response dimension, yields a probability distribution of response values. The result can be described formally as a mixture model, composed of a uniform component (resulting from background activation in the binding space), a distribution reflecting all encoded response features equally (resulting from the uniform component in cue space combined with activation peaks in the binding space), and a component reflecting the encoded response features scaled according to the similarity of the memorized items to the cue feature. Although the effects of interference between items are emphasised in the model, it can be seen that the random noise component - which is assumed to grow with set size - also plays a significant role in response generation. In order to account for effects of cuing on memory performance, the model additionally assumes that a single memory item can be held in a "focus of attention" with higher precision and reduced noise.

Oberauer and Lin (2017) specifically avoided mapping their conceptual binding space to a single neural mechanism, but they give a number of possible neural implementations. One that has already been used in related models for verbal memory (with lists of discrete words instead of continuous visual features, Oberauer et al., 2012) assumes that working memory is based on rapid synaptic plasticity (compare Stokes, 2015), equating the binding space with a weight matrix of synaptic connections between different populations of neurons that represent the individual feature spaces. Alternatively, the authors considered the possibility of realizing the model through a conjunctive population code (as described for the next model). They also tested an implementation variant intended to reflect binding through synchronous spiking activity, with spike phase as an additional intermediate feature space, but found that it provided a poorer fit to experimental data than the original implementation. The model has been used to fit data for set size effects in cued recall tasks, and in particular accounts for different effects of item similarity in the cue and feature dimension.

\section{Conjunctive population code models}

The model of Schneegans and Bays (2017) is conceptually similar to the idea of binding spaces in the interference model, but is explicitly implemented as a neural population model with conjunctive coding. Previous research had shown that decoding from neural population activity with random noise can explain response distributions in cued recall tasks, with normalization of total activity in a population accounting for set size effects (Bays, 2014). In the extended version of this model with conjunctive coding, each neuron in the population is sensitive to both of the features being bound, and the tuning curves of the population as a whole cover the complete space of possible feature conjunctions (Figure 4C). In memorizing an array of visual stimuli, the population response for each stimulus is determined separately based on the neural tuning curves, with additional random noise added. To perform a cued recall task, the cue and response feature values of all memorized items are decoded from the population activity via maximum likelihood estimation. The item whose decoded cue feature value is closest to the given cue is selected, and its decoded response feature is produced as response. The 
model was applied to fit behavioural data from cued recall tasks testing colour-location binding, using either colour or location as a cue in different blocks of trials. The different patterns of response distributions and proportions of swap errors in the two task variants were accurately reproduced by decoding from a single conjunctive population, with different tuning widths for colour and location. The model also explained effects of cue similarity on the probability of swap errors, similar to the interference model.

The population coding model also explicitly addresses binding between more than two features (Figure 4D). In an extension of the basic mechanism, the concept of binding via space is employed to account for cued recall data when sample arrays contain multiple visual features (colour, orientation, and location). To memorize such displays, two conjunctive population codes for two features are combined, one representing colour-location conjunctions and the other orientation-location conjunctions. When cued e.g. with a colour, the model will first estimate the cued item's location via one population code, and then use that location to determine the orientation via the second population. The authors demonstrated that this binding mechanism can explain response correlations in dual-report tasks (e.g. cue with colour, report both location and orientation) significantly better than an alternative version with a direct representation of colour-orientation conjunctions.

One limitation of the model of Schneegans and Bays (2017) is that it employs separate population representations for each item (to make maximum likelihood decoding mathematically feasible). The closely related model of Matthey et al. (2015) superimposes representations of all items in a single conjunctive population representation. This may have greater biological plausibility, but it requires additional assumptions about the decoding mechanism, and makes it computationally infeasible to fit single-trial data. The latter model additionally combines the conjunctive population code with separate population codes for the individual features, to yield a "mixed code" (Figure 5A). This provides a more efficient representation of precise feature values and their bindings. Simulations showed that a proportion of $85 \%$ conjunctive neurons to $15 \%$ single-feature neurons provided a good approximation to experimentally observed proportions of swap errors in cued recall tasks.

Another limitation that holds for all models described so far is that they do not specify a mechanism by which neural activity is sustained during the retention interval. This was addressed in the work of Schneegans et al. (2016), who proposed a detailed neural architecture to autonomously perform the processes of memorization, maintenance, and comparison, and to solve a variety of change detection tasks with different requirements regarding feature binding (Figure 5B). The model is implemented as a set of differential equations describing neural activation dynamics under the influence of external stimulation and internal interactions, with working memory realized as attractor states in this neural dynamics (Wang, 2001). The model employs conjunctive population coding with binding via space, but expands the neural architecture with additional components for visual perception, attentional selection and feature comparison. Consistent with Feature Integration Theory, the model assumes that multiple individual features can be processed and compared simultaneously (using the change detection mechanism of Johnson et al., 2009), making use of parallel feature pathways; but attentional selection of individual items is required to memorize and compare feature conjunctions, which 

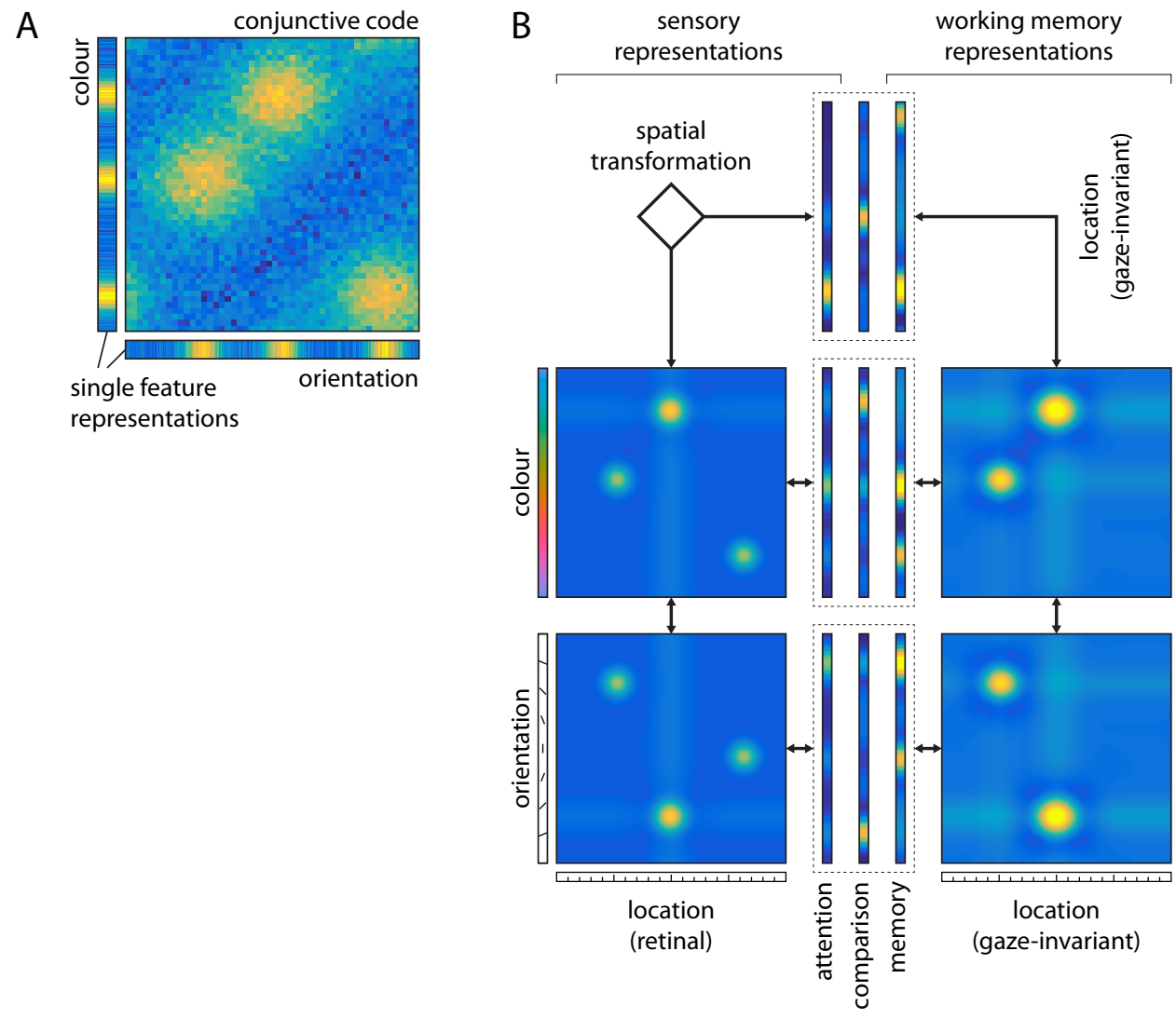

Figure 5: Variants of conjunctive population models. (A) Population model with mixed representation (Matthey et al., 2015), using a combination of conjunctive coding to capture the binding of features (here, colour and orientation for three memorized items) and separate population codes that provide a fine-grained representation of individual feature values. Activity in each population is colour-coded. (B) Neuro-dynamic model to describe the processes of memory formation and change detection, using conjunctive population codes with binding via space (Schneegans et al., 2016). The model employs a sensory level of representations that receives visual input (left, with feature maps over space for colour and orientation), and forms a memory representation of visual items in the form of self-sustained peaks of activation (right, with the same representational format). It employs separate pathways for locations and individual feature spaces at an intermediate level (center), in order to allow an efficient transformation of location information from a retinal to a gaze-invariant reference frame for memorization (top left). This necessitates a sequential process in which items are attended one-by-one for memorization and comparison (shown here for a partially completed memorization of three visual stimuli, with the centrally located item currently selected by visual attention). 
rely on recombining the different feature pathways. However, due its complexity, this model has not been able to quantitatively fit behavioural data.

\section{Model accounts of binding failures}

A consistent feature of the models reviewed here is that their core WM representations are inherently conjunctive, i.e. they encode a combination of features. This is in contrast to the assumption of the Object File Theory that features are stored separately, with additional integrated object files available only for a small number of items. Only two of the models employ separate feature representations as part of working memory, Schneegans et al. (2016) and Matthey et al. (2015), with the latter explicitly investigating their contribution to memory and recall performance.

From a theoretical point of view, such separate representations are attractive in that they provide greater efficiency of representation. This is particularly relevant when using a conjunctive population code to represent combinations of features (Fusi et al., 2016; the case is somewhat less clear for synaptic mechanisms as proposed by Oberauer and Lin, 2017): to adequately cover a conjoined feature space spanned by two feature dimensions with the tuning curves of a neural population, many more neurons are required than to cover the two separate, one-dimensional feature spaces. So if only the individual features and not their bindings are relevant, separate feature representations can provide the same precision using far less neural resources. This effect is employed in the model of Matthey et al. (2015), and forms the basis for their account of swap errors in cued recall tasks: in determining the most likely response value of the cued item from the mixed population code, the decoding of correct binding can fail due to noise in the conjunctive population, while the separate feature representations still allow a reliable estimation of the individual feature values from the sample array.

\section{Swap errors as effect of cue similarity}

How do the models without such mixed representations explain swap errors? The binding pool model does not actually produce true swap errors under the conditions tested by Swan and Wyble (2014), but interference between multiple memorized items produces biases that can be misclassified as swap errors by a mixture model. This mechanism does provide good fits to experimental data in cued recall tasks for colour, but it is less clear whether it can account for findings in location recall, where responses are typically more precise and have been shown to cluster specifically around the actual locations of target and non-target items (Pertzov et al., 2012; Schneegans and Bays, 2016). In contrast, the models of Oberauer and Lin (2017) and Schneegans and Bays (2017) do produce real swap errors centred on the features of a non-target item, and they both do so via the same mechanism: confusions based on feature similarity in the cue dimension (Bays et al., 2009).

Several previous studies had already found support for the notion that swap errors depend on the cue dimension. Rajsic and Wilson (2014) reported that in a cued recall task for coloured rings, the proportion of swap errors and guesses depended on which feature dimension (colour 
or location) was used as the cue and which one had to be reported. This indicates that swap errors do not primarily reflect the forgetting of bindings or whole items, because such forgetting would affect performance equally independent of the cue dimension used. ${ }^{1}$ Additionally, the proportion of swap errors can be reduced when multiple redundant cues are given (Rajsic et al., 2017). Other studies had observed that, when location was used as the cue, swap errors depended on the spatial proximity between items, occurring much more frequently between items that were close to each other (Emrich and Ferber, 2012; Rerko et al., 2014; Bays, 2016; see also Souza et al., 2014).

Both Oberauer and Lin (2017) and Schneegans and Bays (2017) extended these results to other feature dimensions, demonstrating that swap errors occur preferentially for items whose feature value in the cue dimension is similar to the target item (and thus, similar to the given cue) - regardless of whether that cue dimension is location or colour. Both models account for this effect through imprecision in recall of the cue dimension features: in the model of Schneegans and Bays, decoding variability from the noisy population code can lead to a non-target item being selected as the one most similar to the given cue, especially if its cue feature is similar to the target (Figure 4C); and in the model of Oberauer and Lin, overlap of similar items' broad activity bumps in the binding space with the cue input leads to activation of response features for certain non-target items (Figure 4B; in addition, background noise causes a certain proportion of swap errors for all non-targets independent of cue similarity). Both models successfully fit cue similarity effects in quantitative detail. While the conjunctive population model uses notably fewer free parameters to achieve this, no formal model comparison has yet been carried out between these two models.

\section{Implications for change detection tasks}

What do these proposed mechanisms mean for binding deficits in change detection tasks? The binding pool model explicitly addresses change detection, and explains differences in detecting feature and conjunction changes by biases in recalled features towards the features of other memorized items (the same effect that explains swap errors in this model). In addition, its change detection mechanism takes into account the possibility that a correspondence between sample and test item cannot be unambiguously established (based on location), in which case a change in that item remains undetected. The models of Oberauer and Lin (2017) and Schneegans and Bays (2017) do not address change detection directly, but the distributions of response probability they generate for cued recall tasks (shown in Figure 4B and C) nonetheless provide some intuition as to why conjunction changes may be harder to detect than novel features. The probability distributions show peaks around the report features of several non-targets, in addition to the peak around the actual target feature value (as a result of uncertainty with respect to the cued item). If these distributions are used to decide if a given test feature matches the one in memory for a single item, a feature value coinciding with one of these non-target peaks would produce a weaker change signal than a novel feature that

\footnotetext{
${ }^{1}$ It should be noted that Rajsic and Wilson (2014) also found an increase of guess errors if all non-target items were presented again at test, while swap errors essentially disappeared in this condition. This may indicate that some swap errors are the result of forgetting whole items.
} 
does not match any of them (compare Cowan et al., 2006 for a signal detection interpretation of conjunction changes).

While this suggests an intuitive explanation of reduced performance in conjunction change detection, turning it into a concrete implementation is not entirely straightforward. The models discussed here assume that response generation corresponds to drawing a single sample from the probability distribution, without any part having explicit access to the full distribution. This generates swap errors in some trials, but these would only by chance match a specific conjunction change in a change detection trial. Donkin et al. (2015) tested a model that allowed random swaps to occur within a framework based on a fixed number of slots for memory items, and found that it failed to capture empirically observed proportions of hits and false alarms, especially at higher set sizes. A model producing swap errors based on noise in the cue dimension may produce a different pattern of responses than this slot-based approach, but no study has directly tested this, and it remains unknown at this time whether such a mechanism alone can account for differences in detecting feature and conjunction changes.

Another important aspect of the binding problem is to consider whether memorizing bindings and detecting conjunction changes involve sequential or parallel operations. Feature Integration Theory proposed that the formation and comparison of feature bindings requires focused attention, necessitating sequential processing of visual stimuli. The binding pool model likewise assumes that items are memorized and compared in a sequential fashion, independent of the binding requirement in the task. In contrast, in the neural process model of Schneegans et al. (2016), a sequential operation is assumed only for memorization and comparison of feature conjunctions, while the detection of a novel feature is performed fast and in parallel, causing automatic deployment of attention to the change (as observed by Hyun et al., 2009). If a slower sequential process is indeed required to detect recombination of features, this could further contribute to reduced binding change detection, especially for whole-array tests. Moreover, if different modes of operation are required for feature and conjunction changes, a failure to employ the correct mode may explain certain biases in change detection tasks (e.g. false alarms for irrelevant conjunction changes, Treisman and Zhang, 2006; see below). Elucidating the detailed processes underlying change detection is not trivial using behavioural studies (Gilchrist and Cowan, 2014), but must nonetheless form an important part of a full understanding of visual feature binding.

\section{Reconsidering the binding deficit}

Taken together, the findings and theoretical considerations described above lead us to question the assumption that there exists a specific limitation for memorizing feature conjunctions. The occurrence of swap errors in cued recall tasks, and potentially also impaired detection of binding changes in change detection tasks, can be explained by the limited precision for recalling individual features resulting from inherently conjunctive representations. This suggests that there exists a single memory substrate that inherently contains binding information, instead of a number of separate stores for individual features supplemented by a more capacity-limited or more error-prone store for binding between features. While the existence of separate memory stores for individual features remains theoretically attractive, as it would provide high-fidelity 
representations with lower demand for neural resources, it does not appear necessary to explain the limitations of human memory performance.

There is also little direct evidence from neurophysiology for a separation of substrates involved in memorizing features and binding information (a question typically framed as whether there exist binding-specific brain areas). Shafritz et al. (2002) compared fMRI signals in a change detection task requiring memory either for conjunctions or individual features (colour and shape), and found increased activity in the conjunction condition in parietal regions associated with spatial attention. However, it was not tested whether this activity also occurred during the (very brief) retention interval, and the effect was not observed when two sample items were presented sequentially at the same location. Sala and Courtney (2007) did not find any binding-specific activation during the retention interval of a working memory task. A more recent $f M R I$ study did report a variety of cortical regions with increased activity during the retention interval for a conjunction condition compared to single-feature conditions (Parra et al., 2014), although some of these regions overlapped with areas active for single features during encoding. An involvement of the hippocampus for feature binding in working memory has also been proposed, but the evidence is inconclusive (Piekema et al., 2006, 2010; see also section "Role of medial temporal lobe", below).

Are models with primarily conjunctive representations consistent with the evidence described in the first section for largely independent feature stores? First, it must be considered that in these models with noisy, limited-precision representations, errors in encoding and decoding different feature dimensions can still occur independently, and the precision of recall can vary greatly between different feature dimensions even when encoded in a conjunctive code (e.g. due to differences in neural tuning widths, Schneegans and Bays, 2017). More importantly, however, most of these models deal foremost with binding of a single surface feature (e.g. colour) to a location, whereas most findings about feature independence deal with different surface features (colour, orientation, shape, etc.). Two of the models described here that explicitly deal with binding of multiple surface features (Schneegans et al., 2016; Schneegans and Bays, 2017) employ separate population codes for different surface features that are bound only via shared location information. We will review the plausibility of this mechanism and the role of spatial location in binding in the next section.

\section{The role of space and time for binding}

Binding more than two visual features together poses a challenge for conjunctive population codes. Covering the high-dimensional space spanned by multiple features with neural tuning functions quickly becomes infeasible. One possible solution is to employ a specific binding dimension that mediates the binding of all other features. This could be an abstract dimension, as realized in the token nodes of the binding pool model (although in the concrete implementation, these serve for disambiguation in a conjunctive code rather than to directly mediate binding). An alternative is binding via space, as implemented in the models of Schneegans and Bays (2017) and Schneegans et al. (2016): each surface feature is bound via a conjunctive population code to a location, and only the shared location binds different surface features 
to each other. This kind of representation matches the classical concept of feature maps over space (as used in Feature Integration Theory), without requiring an additional representation format like object files. Another possibility is that the presentation time or ordinal position of an item in a sequence has a special role in binding: when items are presented simultaneously the ordinal information could arise from sequentially selecting items with focused attention. We will review experimental evidence and potential problems for these different approaches.

\section{Evidence for a privileged role of space}

We have already addressed the importance of location for the object benefit, and the susceptibility of bindings to interference from tasks that require strong visuo-spatial attention. In general, object location is a salient feature that can be memorized with high precision and is highly effective as a cue for recall of other features (Schneegans and Bays, 2017). EEG studies show that spatial attention can be drawn to items in working memory (Griffin and Nobre, 2003), even when the cue is non-spatial and location is entirely irrelevant for the task (Kuo et al., 2009; DellAcqua et al., 2010; Eimer and Kiss, 2010). The task-irrelevant location of a stimulus can also be directly decoded from EEG data (Foster et al., 2017). These findings and consistent behavioural results (Theeuwes et al., 2011) indicate that location is automatically encoded and reflected in sustained neural activity during working memory delays (see also Olson and Marshuetz, 2005; Elsley and Parmentier, 2015).

Direct behavioural evidence for a role of location in binding other features was presented in the influential study of Treisman and Zhang (2006) (recently replicated by Udale et al., 2017). They showed, first, that in a change detection task for novel features, task-irrelevant changes in binding of colour and shape were often misreported as feature changes when the items in sample and test array were presented at the same locations, but not when items were presented at new locations (Figure 1C); and second, that when the task required detecting changes in shape-colour binding, performance was better when the sample and test arrays were presented at the same than at different locations. The authors argued that binding between colour and shape is at least in part mediated by their shared location. They also noted that it is likely not absolute object location that is relevant for these effects, but relative location within a spatial configuration of objects, as supported by differences between single-item and whole array probe conditions in their experiments, and a number of other studies (Jiang et al., 2000; Hollingworth, 2007; Hollingworth and Rasmussen, 2010).

A similar conclusion about feature binding was reached by Saiki and Miyatsuji (2007). They used a cyclically occluded array of coloured shapes, and compared detection performance for swapping colours, shapes, or whole items between two locations. The results indicated that initial detection of swaps is based on memorized bindings of individual features to locations, although once attention is drawn to a specific item by a first swap, a more integrated representation of all features can be used to identify further changes. Kondo and Saiki (2012) further confirmed the finding that randomly changing items' locations interfered with the detection of changes in colour-shape binding, while random changes in the colours or shapes did not interfere with monitoring bindings of the other feature to location.

One possible limitation to the findings supporting a privileged role of space is that most 
studies used relatively short retention intervals of around one second (Schneegans and Bays, 2017; Kondo and Saiki, 2012). This is long enough to rule out short-lived iconic (sensory) memory as a basis for recall performance in these tasks. However, there is evidence that the effects of task-irrelevant location changes on change detection performance for feature bindings decline significantly over longer delay durations (Treisman and Zhang, 2006; Logie et al., 2011; see also Bocincova et al., 2017). It is therefore possible that location is important for feature binding only at short to intermediate delays, and a different storage format supports memory at longer delays.

\section{Space as a binding dimension}

Schneegans and Bays (2017) tested the hypothesis that non-spatial features are only bound via their location using fits of their conjunctive population model to behavioural data. They used a dual-report task, in which subjects were cued with either colour or orientation and reported both the other non-spatial feature and the location of the cued item from the sample array. Errors in both reports were strongly correlated, and the feature report was centred on the feature at the reported location (even if that location was wrong). This result contrasts with the finding of independent (uncorrelated) recall error for different non-spatial features described earlier (Bays et al., 2011; Fougnie and Alvarez, 2011; Fougnie et al., 2013), but both are consistent with a spatial binding account: if a spatial cue is used to retrieve two non-spatial features, these are retrieved from different feature-location representations, in which maintenance or retrieval can fail independently. But if one non-spatial feature is used as a cue to retrieve another one bound to it via a shared location, a failure to retrieve the location will necessarily result in a failure to retrieve the other feature. This reasoning follows the much earlier study of Nissen (1985), and matches analogous findings for perceptual binding (Bundesen et al., 2003; Vul and Rich, 2010; Huang, 2010).

The interpretation of binding via space is also supported by the study of Rajsic et al. (2017). They demonstrated that multiple redundant cues led to better recall performance, but that the effect was strongest when two non-spatial features (colour and orientation) were used to retrieve a location. Adding another non-spatial feature to a spatial cue had little to no effect as expected if that additional feature could only be used to retrieve the item location already provided. The disruptive effect of spatial scrambling of the test array in a change detection task (Treisman and Zhang, 2006; Kondo and Saiki, 2012) can be explained as depriving observers of the use of a spatial cue to directly access and compare the features of each memory item (Levillain and Flombaum, 2012; but see also Gilchrist and Cowan, 2014), forcing them instead to use e.g. the shape of an item to indirectly access its colour via their shared location in memory. Related to this, it has been found that adding spatial structure to a memory display improves children's performance for detecting binding changes (Simmering and Wood, 2017). The concept of binding via space is also plausible from a neural perspective, given that most neurons involved in perceptual or memory representations of visual features are found to also exhibit spatial selectivity (Op De Beeck and Vogels, 2000; Rao et al., 1997).

Arguing against a necessary role of location for memorizing visual sample arrays, Woodman et al. (2012) reported that performance in a colour change detection task was unimpaired 
under various types of task-irrelevant location and size changes between sample and test. Notably however, this task did not require any binding of features (and see Levillain and Flombaum, 2012 for further limitations). Saiki (2016) argued that a location-independent storage mechanism exists for colour-shape bindings, in addition to a mechanism that binds features to locations. In their experiment, participants had to detect any feature match between a single probe item and either of two sample items. They found a reaction time benefit when the probe matched two features from the same item, independent of location match between sample and probe. However, such a single-item benefit could still arise when features are bound via space, if attentional selection in working memory tends to activate whole spatially-bound items even when cued with a non-spatial feature (as the results of Kuo et al., 2009 and others suggest). The feasibility of such a mechanism has been demonstrated in the neural model of Schneegans et al. (2016), to address detection of conjunction changes when locations are scrambled.

One challenge for a system that employs binding via space is how to deal with object movement (indeed, some have argued that for this reason location should be considered an extrinsic object property that is less strongly bound than intrinsic properties like colour and shape, see Parra, 2017). Hollingworth and Rasmussen (2010) investigated this by briefly presenting colours within squares, then moving the empty squares around during the retention interval. They found that the colours were primarily bound to the locations where they were originally presented, although some transfer to the new locations occurred (see also Bapat et al., 2017). A further problem is how to deal with eye movements, which shift the whole visual field. The model of Schneegans et al. (2016) employs a transformation of object location into a gazeinvariant reference frame to keep the representation stable, but some recent findings suggest a retinotopic reference frame for WM (Shafer-Skelton et al., 2017). Feature-location binding can be disrupted at the time of a gaze change (Golomb et al., 2014), but to our knowledge no study has empirically tested the effects of gaze shifts on binding between non-spatial features.

\section{Binding in time}

The grouping of individual visual features into objects can be defined not only by their spatial arrangement, but also by temporal conjunctions. As described earlier, presenting items sequentially at the same location has been found to result in impaired change detection performance for binding compared to the simultaneous presentation of a sample array (Allen et al., 2006). Gorgoraptis et al. (2011) showed that the sequential presentation of items in a cued recall task leads to an increase in the proportion of swap errors compared to simultaneous presentation. Kool et al. (2014) found that swap errors for sequentially presented items in a recall task with spatial cue and colour report mostly involved features earlier in the sequence being replaced by features of later items, consistent with an overall recency effect. Pertzov and Husain (2014) directly investigated whether location plays a role in binding features of sequentially presented items, by showing coloured oriented bars sequentially either at the same or at different locations. When participants were cued with a colour to report an item's orientation, they made significantly more swap errors in the same-location condition, supporting a role of location in binding even when it is task-irrelevant (but see Harrison and Bays, 2018 for a failure 
to find this effect when systematically varying stimulus distances).

Despite these limitations, humans clearly can still retain bindings for objects presented sequentially at the same location, as evidenced by above-chance performance in the tasks described above. EEG studies have found equal amplitudes of the CDA when items are presented at the same or at different locations (Ikkai et al., 2010). Likewise, fMRI studies have shown some regions that are sensitive only to feature load, independent of presentation mode, while other areas seem to respond to the number of different item locations in a task (Xu and Chun, 2006). Within spatial binding models, one possibility highlighted by Schneegans and Bays (2017) is that items presented at the same location could be internally remapped to unoccupied locations to avoid misbindings, consistent with the proposal of Abrahamse et al. (2014).

The interference model of Oberauer and Lin (2017) does not directly address binding for sequentially presented items, but does not explicitly rely on space for binding other features, either. Closely related earlier models for verbal working memory employ ordinal position as a context dimension to which a representation of each word is bound, such that the order of memorized words is retained (Oberauer et al., 2012). Transposition errors in serial recall (swap errors in the order of items) occur in the model due to cue similarity effects in the ordinal dimension (see also Brown et al., 2007). In principle, different forms of binding to location, sequential position, or among different visual surface features may coexist in this type of model, but their relationship has not been specified.

The binding pool model of Swan and Wyble (2014) takes a somewhat complementary view to the spatial binding model of Schneegans and Bays (2017), emphasizing the role of sequential order rather than space. In particular, the binding of features to tokens and the comparison between memorized sample and test arrays are assumed to always occur in a sequential fashion even for simultaneously presented stimulus arrays. Moreover, earlier implementations of the model addressed the encoding of visual stimuli in a rapid serial visual presentation task (Bowman and Wyble, 2007), with the sequential position information encoded through binding of visual features to ordered token nodes. This model accounted for the attentional blink effect under varying conditions. It also addressed a kind of swap error in which stimulus order is misreported when two sample items appear in rapid succession, which is explained by erroneous binding of two items to the same token node (Wyble et al., 2009, 2011).

While memory for sequential order has been intensely studied both in verbal and visual working memory (Hurlstone et al., 2014), and sequential position is regularly used as a retrieval cue in working memory tasks (Harrison and Tong, 2009), its relationship to binding between other features is not well understood. Time or ordinal position may take a similar role as proposed for location in spatial binding models, perhaps combined with it in a spatiotemporal binding dimension, but further research is needed to clarify the underlying principles.

\section{Binding in ageing and clinical conditions}

The effects of both healthy ageing and a number of clinical conditions on the ability to memorize and recall feature conjunctions has been intensely studied. This has been motivated at least 
in part by findings from long term memory research, where decline of memory performance with age has been found to particularly affect relational and associative memory (Old and Naveh-Benjamin, 2008). Specific deficits in working memory performance might help to further elucidate the mechanisms of binding, and they could also serve as a diagnostic tool for clinical conditions.

Binding deficits in working memory have been assessed by comparing memory performance of older adults or patients with control groups, most often using change detection tasks (and sometimes verbal report tasks) that separately test memory for single features and feature conjunctions. It should be noted that interpretation of these results suffers from the same limitations as discussed above in section "Problems of assessing the binding deficit". An additional problem arises when comparing populations that have decreased memory performance in general: If the detection of a conjunction change depends on accurately retrieving two features of a memorized object (e.g. using one as a retrieval cue and the other for comparison), an independent decrease in memory precision for each feature can lead to a larger compound deficit in detecting binding changes. Analogously, in a binary view of memorizing features, a reduced probability of independently memorizing each feature of an object will lead to a larger decrease in the probability of remembering multiple features of the same object (Cowan et al., 2013).

\section{Effects of healthy ageing}

For conjunctions of different surface features (such as colour and shape), a host of studies has found little indication of a specific decrement of binding memory with age, even though overall memory performance is generally worse in older than younger adults (Brockmole et al., 2008; Parra et al., 2009b; Isella et al., 2015; Read et al., 2016; Hoefeijzers et al., 2017; with conflicting results only in a single experiment in Brown and Brockmole, 2010). In contrast, some earlier studies did report a specific decrement in performance for tasks requiring binding of features or objects to location (Mitchell et al., 2000b,a; Cowan et al., 2006; see also Brockmole and Logie, 2013). Such a deficit in feature-location binding without corresponding effects on featurefeature binding would be in conflict with a role of space in binding other features. However, the results of these studies might be explained by a combination of memory deficits in the individual features (as described in the previous section), and in any case they have not been replicated in subsequent studies (Read et al., 2016; Rhodes et al., 2017; the latter also discusses further issues with the earlier experiments). Effects of ageing on feature-location binding have also been investigated using a cued recall task, finding both a decrease in response precision and an increase in the proportion of swap errors for older adults (Peich et al., 2013). A subsequent study found that an observed increase in swap errors for object-location binding disappeared when taking into account the decreased accuracy of object identification, which is consistent with the view that binding memory is not selectively affected by ageing (Pertzov et al., 2015).

While most of these studies relied on statistical null results to support the absence of specific binding deficits, several newer studies have used Bayesian statistics to address the same question. Confirming the interpretation of previous results, these studies have consistently found evidence against a specific effect of age on binding memory for either feature-feature or 
feature-location binding (Rhodes et al., 2017), independent of presentation format and duration of the sample array (Rhodes et al., 2016; Brown et al., 2017). We also note in this context that during infant development, memory for feature-location binding seems to emerge at the same time as multi-item WM (Oakes et al., 2006).

\section{Role of medial temporal lobe}

The medial temporal lobe, and particularly the hippocampus, has long been considered to play a central role in long term memory and the learning of different kinds of associations (Mayes et al., 2007). It has more recently also been implicated in working memory functions and binding in particular. Mitchell et al. (2000a) found decreased hippocampal activity in older compared to younger adults specifically in the object-location binding condition of a WM task, along with a specific binding deficit in behavioural performance (which is not however consistent with the larger literature, see above). In studies of patients with temporal lobe damage, Olson et al. (2006) found a deficit in object-location binding following longer, but not shorter retention intervals, and Pertzov et al. (2013) reported an increase in swap errors in a cued recall task for locations. However, Baddeley et al. (2010) did not observe any binding deficit in a single case study of a patient with hippocampus damage, and for a different patient Parra et al. (2015a) found a deficit in relational binding (i.e., binding between features or objects that are presented separately from each other), but not conjunctive feature binding. In fMRI studies with healthy subjects, Piekema et al. (2006) found support for the involvement of the hippocampus in object-location binding, but a subsequent experiment indicated that the hippocampus only has a role in relational binding between objects (Piekema et al., 2010; see also Hannula and Ranganath, 2008; Parra et al., 2014). These conflicting findings do not allow a conclusive assessment of the medial temporal lobe's role in binding features in WM at this time.

\section{Binding in Alzheimer's disease}

While specific binding deficits in WM have remained elusive or inconclusive in other populations, one clinical condition for which a deficit in feature binding appears strongly supported by behavioural data is Alzheimer's Disease (AD). A specific and often substantial decrement in performance has been observed in change detection tasks requiring object-colour (Parra et al., 2009a) and colour-colour binding (which may be mediated by colour-location binding; Parra et al., 2011b), as well as in binding for objects to locations in cued recall tasks (Liang et al., 2016). The deficit occurs in both sporadic AD and familial AD caused by a specific genetic mutation, as well as in asymptomatic carriers of the mutation (Parra et al., 2010b; Liang et al., 2016). This binding deficit has been proposed as a diagnostic tool since it allows differentiation between AD and other forms of dementia and clinical conditions (Parra et al., 2010a; Della Sala et al., 2012; Cecchini et al., 2017; see also Parra, 2017; Liang et al., 2017).

Several studies have also related these behavioural signatures to physiological and functional changes in the brain. The magnitude of the binding deficit in familial AD patients and asymptomatic gene carriers has been observed to correlate with measures of white matter integrity (Parra et al., 2015b) and hippocampus volume (Liang et al., 2016). While such findings 
might suggest that the deficits in $A D$ are caused by damage to specific structures that mediate binding memory (separately from feature memory), an alternative explanation is that $A D$ causes a disruption of the processes required to form or retrieve bindings. This view is supported by a recent EEG study that found changes in event-related potentials during WM tasks in $A D$ and other patients, which were tentatively interpreted as an impairment of attentional control during the encoding phase (Pietto et al., 2016).

\section{Conclusions}

The successful retention of feature bindings has as a prerequisite the storage of two (or more) individual features. If we accept that these features are memorized in a noisy form that limits the precision of recall and comparison, then detecting a change in the conjunction of features, or retrieving a feature based on a different feature of the same object given as a cue, inherently involves additional computation and more potential sources of error compared to recalling the individual features independent of their binding. Therefore, failures of binding recall do not necessarily constitute evidence that memorizing conjunctions requires additional memory resources or mechanisms that are not employed in memory for single features. We believe that taking this into account, there is currently no compelling reason to postulate a separate substrate for memory of binding, although research that takes into account the uncertainty in each individual feature dimension might in future provide evidence for this view. A more open question is whether distinct processes are involved in encoding and comparing feature conjunctions compared to unbound features, in particular for change detection. Further empirical and theoretical research is needed to answer this question.

We further believe that the traditional distinction of storing integrated objects versus individual features is too simplistic. While all working memory models discussed here employ some form of conjunctive representation, these are realized in a distributed fashion (in particular for binding between more than two feature dimensions), rather than as self-contained units for each bound object. These distributed representations allow for errors in recall for features and conjunctions to occur at different stages, and different aspects of the representation may be disrupted independently. Future research must focus more on how specific conjunctions are formed and maintained in memory, rather than on a binary distinction between conjunction and feature memory.

Finally, we find that one of the most basic formats proposed for the neural representation of visual items - feature maps over visual space - successfully accounts for a large range of empirical findings, without needing to postulate additional representational structures like object files. Feature maps are consistent with the evidence for largely independent memory stores for different feature dimensions, while still allowing for storage and retrieval of feature conjunctions by extracting the features at a single location from different maps. Future theoretical accounts of binding need to be assessed based on the advances they provide over this mechanism, and empirical research should explore whether this account is indeed sufficient to explain human performance in memory for feature binding. 


\section{Acknowledgements}

This work was supported by the Wellcome Trust (grant no. 106926).

\section{References}

Abrahamse, E., Van Dijck, J.-P., Majerus, S., and Fias, W. (2014). Finding the answer in space: the mental whiteboard hypothesis on serial order in working memory. Frontiers in Human Neuroscience, 8:932.

Allen, R. J., Baddeley, A. D., and Hitch, G. J. (2006). Is the binding of visual features in working memory resource-demanding? Journal of Experimental Psychology: General, 135(2):298.

Allen, R. J., Baddeley, A. D., and Hitch, G. J. (2014). Evidence for two attentional components in visual working memory. Journal of Experimental Psychology: Learning, Memory, and Cognition, 40(6):1499.

Allen, R. J., Castellà, J., Ueno, T., Hitch, G. J., and Baddeley, A. D. (2015). What does visual suffix interference tell us about spatial location in working memory? Memory \& cognition, 43(1):133-142.

Allen, R. J., Hitch, G. J., and Baddeley, A. D. (2009). Cross-modal binding and working memory. Visual Cognition, 17(1-2):83-102.

Allen, R. J., Hitch, G. J., Mate, J., and Baddeley, A. D. (2012). Feature binding and attention in working memory: A resolution of previous contradictory findings. Quarterly journal of experimental psychology, 65(12):2369-2383.

Alvarez, G. A. and Thompson, T. W. (2009). Overwriting and rebinding: Why feature-switch detection tasks underestimate the binding capacity of visual working memory. Visual Cognition, 17(1-2):141-159.

Awh, E., Barton, B., and Vogel, E. K. (2007). Visual working memory represents a fixed number of items regardless of complexity. Psychological science, 18(7):622-628.

Axmacher, N., Henseler, M. M., Jensen, O., Weinreich, I., Elger, C. E., and Fell, J. (2010). Cross-frequency coupling supports multi-item working memory in the human hippocampus. Proceedings of the National Academy of Sciences, page 200911531.

Baddeley, A. (2000). The episodic buffer: a new component of working memory? Trends in cognitive sciences, 4(11):417-423.

Baddeley, A., Allen, R., and Vargha-Khadem, F. (2010). Is the hippocampus necessary for visual and verbal binding in working memory? Neuropsychologia, 48(4):1089-1095.

Baddeley, A. D. and Hitch, G. (1974). Working memory. In Psychology of learning and motivation, volume 8, pages 47-89. Elsevier. 
Bapat, A. N., Shafer-Skelton, A., Kupitz, C. N., and Golomb, J. D. (2017). Binding object features to locations: Does the spatial congruency bias update with object movement? Attention, Perception, \& Psychophysics, 79(6):1682-1694.

Bays, P. M. (2014). Noise in neural populations accounts for errors in working memory. Journal of Neuroscience, 34(10):3632-3645.

Bays, P. M. (2016). Evaluating and excluding swap errors in analogue tests of working memory. Scientific reports, 6 .

Bays, P. M., Catalao, R. F., and Husain, M. (2009). The precision of visual working memory is set by allocation of a shared resource. Journal of Vision, 9(10):7-7.

Bays, P. M. and Husain, M. (2008). Dynamic shifts of limited working memory resources in human vision. Science, 321(5890):851-854.

Bays, P. M., Wu, E. Y., and Husain, M. (2011). Storage and binding of object features in visual working memory. Neuropsychologia, 49(6):1622-1631.

van den Berg, R., Shin, H., Chou, W.-C., George, R., and Ma, W. J. (2012). Variability in encoding precision accounts for visual short-term memory limitations. Proceedings of the National Academy of Sciences, 109(22):8780-8785.

Bocincova, A., van Lamsweerde, A. E., and Johnson, J. S. (2017). The role of top-down suppression in mitigating the disruptive effects of task-irrelevant feature changes in visual working memory. Memory \& cognition, 45(8):1411-1422.

Bowman, H. and Wyble, B. (2007). The simultaneous type, serial token model of temporal attention and working memory. Psychological review, 114(1):38.

Brady, T. F., Konkle, T., and Alvarez, G. A. (2011). A review of visual memory capacity: Beyond individual items and toward structured representations. Journal of vision, 11(5):4-4.

Brockmole, J. R. and Logie, R. H. (2013). Age-related change in visual working memory: a study of 55,753 participants aged 8-75. Frontiers in psychology, 4:12.

Brockmole, J. R., Parra, M. A., Della Sala, S., and Logie, R. H. (2008). Do binding deficits account for age-related decline in visual working memory? Psychonomic Bulletin \& Review, 15(3):543-547.

Brown, G. D., Neath, I., and Chater, N. (2007). A temporal ratio model of memory. Psychological review, 114(3):539.

Brown, L. A. and Brockmole, J. R. (2010). The role of attention in binding visual features in working memory: Evidence from cognitive ageing. Quarterly Journal of Experimental Psychology, 63(10):2067-2079.

Brown, L. A., Niven, E. H., Logie, R. H., Rhodes, S., and Allen, R. J. (2017). Visual feature binding in younger and older adults: Encoding and suffix interference effects. Memory, 25(2):261-275. 
Bundesen, C., Kyllingsbæk, S., and Larsen, A. (2003). Independent encoding of colors and shapes from two stimuli. Psychonomic Bulletin \& Review, 10(2):474-479.

Cecchini, M. A., Yassuda, M. S., Bahia, V. S., de Souza, L. C., Guimarães, H. C., Caramelli, P., Carthery-Goulart, M. T., Patrocínio, F., Foss, M. P., Tumas, V., et al. (2017). Recalling feature bindings differentiates alzheimers disease from frontotemporal dementia. Journal of neurology, 264(10):2162-2169.

Cowan, N., Blume, C. L., and Saults, J. S. (2013). Attention to attributes and objects in working memory. Journal of Experimental Psychology: Learning, Memory, and Cognition, 39(3):731.

Cowan, N., Naveh-Benjamin, M., Kilb, A., and Saults, J. S. (2006). Life-span development of visual working memory: When is feature binding difficult? Developmental psychology, 42(6):1089.

Davis, G. and Holmes, A. (2005). The capacity of visual short-term memory is not a fixed number of objects. Memory \& cognition, 33(2):185-195.

Della Sala, S., Parra, M. A., Fabi, K., Luzzi, S., and Abrahams, S. (2012). Short-term memory binding is impaired in ad but not in non-ad dementias. Neuropsychologia, 50(5):833-840.

DellAcqua, R., Sessa, P., Toffanin, P., Luria, R., and Jolicœur, P. (2010). Orienting attention to objects in visual short-term memory. Neuropsychologia, 48(2):419-428.

Delvenne, J.-F. and Bruyer, R. (2004). Does visual short-term memory store bound features? Visual cognition, 11(1):1-27.

Delvenne, J.-F. and Bruyer, R. (2006). A configural effect in visual short-term memory for features from different parts of an object. Quarterly Journal of Experimental Psychology, 59(9):1567-1580.

Delvenne, J.-F., Cleeremans, A., and Laloyaux, C. (2010). Feature bindings are maintained in visual short-term memory without sustained focused attention. Experimental Psychology, 57(2):108.

Donkin, C., Tran, S. C., and Le Pelley, M. (2015). Location-based errors in change detection: A challenge for the slots model of visual working memory. Memory \& cognition, 43(3):421-431.

Ecker, U. K., Maybery, M., and Zimmer, H. D. (2013). Binding of intrinsic and extrinsic features in working memory. Journal of Experimental Psychology: General, 142(1):218.

Eimer, M. and Kiss, M. (2010). An electrophysiological measure of access to representations in visual working memory. Psychophysiology, 47(1):197-200.

Elsley, J. V. and Parmentier, F. B. (2015). The asymmetry and temporal dynamics of incidental letter-location bindings in working memory. The Quarterly Journal of Experimental Psychology, 68(3):433-441.

Emrich, S. M. and Ferber, S. (2012). Competition increases binding errors in visual working memory. Journal of Vision, 12(4):12-12. 
Engel, A. K. and Singer, W. (2001). Temporal binding and the neural correlates of sensory awareness. Trends in cognitive sciences, 5(1):16-25.

Foster, J. J., Bsales, E. M., Jaffe, R. J., and Awh, E. (2017). Alpha-band activity reveals spontaneous representations of spatial position in visual working memory. Current Biology, 27(20):3216-3223.

Fougnie, D. and Alvarez, G. A. (2011). Object features fail independently in visual working memory: Evidence for a probabilistic feature-store model. Journal of Vision, 11(12):3-3.

Fougnie, D., Asplund, C. L., and Marois, R. (2010). What are the units of storage in visual working memory? Journal of vision, 10(12):27-27.

Fougnie, D., Cormiea, S. M., and Alvarez, G. A. (2013). Object-based benefits without objectbased representations. Journal of Experimental Psychology: General, 142(3):621.

Fougnie, D. and Marois, R. (2009). Attentive tracking disrupts feature binding in visual working memory. Visual cognition, 17(1-2):48-66.

Franconeri, S. L., Alvarez, G. A., and Cavanagh, P. (2013). Flexible cognitive resources: competitive content maps for attention and memory. Trends in cognitive sciences, 17(3):134-141.

Fusi, S., Miller, E. K., and Rigotti, M. (2016). Why neurons mix: high dimensionality for higher cognition. Current opinion in neurobiology, 37:66-74.

Gajewski, D. A. and Brockmole, J. R. (2006). Feature bindings endure without attention: Evidence from an explicit recall task. Psychonomic Bulletin \& Review, 13(4):581-587.

Gao, T., Gao, Z., Li, J., Sun, Z., and Shen, M. (2011). The perceptual root of object-based storage: An interactive model of perception and visual working memory. Journal of Experimental Psychology: Human Perception and Performance, 37(6):1803.

Gao, Z., Wu, F., Qiu, F., He, K., Yang, Y., and Shen, M. (2017). Bindings in working memory: The role of object-based attention. Attention, Perception, \& Psychophysics, 79(2):533-552.

Gilchrist, A. L. and Cowan, N. (2014). A two-stage search of visual working memory: Investigating speed in the change-detection paradigm. Attention, Perception, \& Psychophysics, 76(7):2031-2050.

Golomb, J. D., LHeureux, Z. E., and Kanwisher, N. (2014). Feature-binding errors after eye movements and shifts of attention. Psychological science, 25(5):1067-1078.

Gorgoraptis, N., Catalao, R. F., Bays, P. M., and Husain, M. (2011). Dynamic updating of working memory resources for visual objects. Journal of Neuroscience, 31(23):8502-8511.

Griffin, I. C. and Nobre, A. C. (2003). Orienting attention to locations in internal representations. Journal of cognitive neuroscience, 15(8):1176-1194.

Hannula, D. E. and Ranganath, C. (2008). Medial temporal lobe activity predicts successful relational memory binding. Journal of Neuroscience, 28(1):116-124. 
Hardman, K. O. and Cowan, N. (2015). Remembering complex objects in visual working memory: Do capacity limits restrict objects or features? Journal of Experimental Psychology: Learning, Memory, and Cognition, 41(2):325.

Harris, A. Z. and Gordon, J. A. (2015). Long-range neural synchrony in behavior. Annual review of neuroscience, 38:171-194.

Harrison, S. A. and Tong, F. (2009). Decoding reveals the contents of visual working memory in early visual areas. Nature, 458(7238):632.

Harrison, W. J. and Bays, P. M. (2018). Visual working memory is independent of the cortical spacing between memoranda. Journal of Neuroscience, 38(12):3116-3123.

Hoefeijzers, S., González Hernández, A., Magnolia Rios, A., and Parra, M. A. (2017). Feature binding of common everyday items is not affected by age. Frontiers in aging neuroscience, $9: 122$.

Hollingworth, A. (2007). Object-position binding in visual memory for natural scenes and object arrays. Journal of Experimental Psychology: Human Perception and Performance, 33(1):31.

Hollingworth, A. and Rasmussen, I. P. (2010). Binding objects to locations: The relationship between object files and visual working memory. Journal of Experimental Psychology: Human Perception and Performance, 36(3):543.

Huang, L. (2010). Characterizing the nature of visual conscious access: The distinction between features and locations. Journal of vision, 10(10):24-24.

Hurlstone, M. J., Hitch, G. J., and Baddeley, A. D. (2014). Memory for serial order across domains: An overview of the literature and directions for future research. Psychological bulletin, 140(2):339.

Hyun, J.-s., Woodman, G. F., Vogel, E. K., Hollingworth, A., and Luck, S. J. (2009). The comparison of visual working memory representations with perceptual inputs. Journal of Experimental Psychology: Human Perception and Performance, 35(4):1140.

Ikkai, A., McCollough, A. W., and Vogel, E. K. (2010). Contralateral delay activity provides a neural measure of the number of representations in visual working memory. Journal of neurophysiology, 103(4):1963-1968.

Irwin, D. E. and Andrews, R. V. (1996). Integration and accumulation of information across saccadic eye movements. Attention and performance XVI: Information integration in perception and communication, 16:125-155.

Isella, V., Molteni, F., Mapelli, C., and Ferrarese, C. (2015). Short term memory for single surface features and bindings in ageing: A replication study. Brain and cognition, 96:38-42.

Jiang, Y., Olson, I. R., and Chun, M. M. (2000). Organization of visual short-term memory. Journal of Experimental Psychology: Learning, Memory, and Cognition, 26(3):683. 
Johnson, J. S., Hollingworth, A., and Luck, S. J. (2008). The role of attention in the maintenance of feature bindings in visual short-term memory. Journal of Experimental Psychology: Human Perception and Performance, 34(1):41.

Johnson, J. S., Spencer, J. P., and Schöner, G. (2009). A layered neural architecture for the consolidation, maintenance, and updating of representations in visual working memory. Brain research, 1299:17-32.

Kahneman, D., Treisman, A., and Gibbs, B. J. (1992). The reviewing of object files: Objectspecific integration of information. Cognitive psychology, 24(2):175-219.

Karlsen, P. J., Allen, R. J., Baddeley, A. D., and Hitch, G. J. (2010). Binding across space and time in visual working memory. Memory \& Cognition, 38(3):292-303.

Kondo, A. and Saiki, J. (2012). Feature-specific encoding flexibility in visual working memory. PLOS One, 7(12):e50962.

Kool, W., Conway, A. R., and Turk-Browne, N. B. (2014). Sequential dynamics in visual shortterm memory. Attention, Perception, \& Psychophysics, 76(7):1885-1901.

Kuo, B.-C., Rao, A., Lepsien, J., and Nobre, A. C. (2009). Searching for targets within the spatial layout of visual short-term memory. Journal of Neuroscience, 29(25):8032-8038.

Lee, D. and Chun, M. M. (2001). What are the units of visual short-term memory, objects or spatial locations? Perception \& Psychophysics, 63(2):253-257.

Levillain, F. and Flombaum, J. I. (2012). Correspondence problems cause repositioning costs in visual working memory. Visual Cognition, 20(6):669-695.

Liang, Y., Pertzov, Y., Nicholas, J. M., Henley, S., Crutch, S., Woodward, F., and Husain, M. (2017). Visual short-term memory binding deficits in alzheimer's disease: a reply to parra's commentary. Cortex, 88:201-204.

Liang, Y., Pertzov, Y., Nicholas, J. M., Henley, S. M., Crutch, S., Woodward, F., Leung, K., Fox, N. C., and Husain, M. (2016). Visual short-term memory binding deficit in familial alzheimer's disease. cortex, 78:150-164.

Logie, R. H., Brockmole, J. R., and Jaswal, S. (2011). Feature binding in visual short-term memory is unaffected by task-irrelevant changes of location, shape, and color. Memory \& Cognition, 39(1):24-36.

Luck, S. J. and Vogel, E. K. (1997). The capacity of visual working memory for features and conjunctions. Nature, 390(6657):279-281.

Lundqvist, M., Rose, J., Herman, P., Brincat, S. L., Buschman, T. J., and Miller, E. K. (2016). Gamma and beta bursts underlie working memory. Neuron, 90(1):152-164.

Luria, R. and Vogel, E. K. (2011). Shape and color conjunction stimuli are represented as bound objects in visual working memory. Neuropsychologia, 49(6):1632-1639. 
Ma, W. J., Husain, M., and Bays, P. M. (2014). Changing concepts of working memory. Nature neuroscience, $17(3): 347$.

Magnussen, S. and Greenlee, M. W. (1999). The psychophysics of perceptual memory. Psychological research, 62(2-3):81-92.

von der Malsburg, C. (1999). The what and why of binding. Neuron, 24(1):95-104.

Marshall, L. and Bays, P. M. (2013). Obligatory encoding of task-irrelevant features depletes working memory resources. Journal of vision, 13(2):21-21.

Matthey, L., Bays, P. M., and Dayan, P. (2015). A probabilistic palimpsest model of visual short-term memory. PLOS Computational Biology, 11(1):e1004003.

Mayes, A., Montaldi, D., and Migo, E. (2007). Associative memory and the medial temporal lobes. Trends in cognitive sciences, 11(3):126-135.

Mitchell, K. J., Johnson, M. K., Raye, C. L., and DEsposito, M. (2000a). fmri evidence of agerelated hippocampal dysfunction in feature binding in working memory1. Cognitive brain research, 10(1-2):197-206.

Mitchell, K. J., Johnson, M. K., Raye, C. L., Mather, M., and D'esposito, M. (2000b). Aging and reflective processes of working memory: Binding and test load deficits. Psychology and aging, 15(3):527.

Morey, C. C. and Bieler, M. (2013). Visual short-term memory always requires general attention. Psychonomic Bulletin \& Review, 20(1):163-170.

Nissen, M. J. (1985). Accessing features and objects: Is location special. Attention and Performance XI, pages 205-219.

Oakes, L. M., Ross-Sheehy, S., and Luck, S. J. (2006). Rapid development of feature binding in visual short-term memory. Psychological Science, 17(9):781-787.

Oberauer, K. and Eichenberger, S. (2013). Visual working memory declines when more features must be remembered for each object. Memory \& Cognition, 41(8):1212-1227.

Oberauer, K., Lewandowsky, S., Farrell, S., Jarrold, C., and Greaves, M. (2012). Modeling working memory: An interference model of complex span. Psychonomic bulletin \& review, 19(5):779-819.

Oberauer, K. and Lin, H.-Y. (2017). An interference model of visual working memory. Psychological review, 124(1):21.

Old, S. R. and Naveh-Benjamin, M. (2008). Differential effects of age on item and associative measures of memory: a meta-analysis. Psychology and aging, 23(1):104.

Olson, I. R. and Jiang, Y. (2002). Is visual short-term memory object based? rejection of the strong-object hypothesis. Perception \& psychophysics, 64(7):1055-1067. 
Olson, I. R. and Marshuetz, C. (2005). Remembering what brings along where in visual working memory. Perception \& Psychophysics, 67(2):185-194.

Olson, I. R., Page, K., Moore, K. S., Chatterjee, A., and Verfaellie, M. (2006). Working memory for conjunctions relies on the medial temporal lobe. Journal of Neuroscience, 26(17):45964601.

Op De Beeck, H. and Vogels, R. (2000). Spatial sensitivity of macaque inferior temporal neurons. Journal of Comparative Neurology, 426(4):505-518.

O'Reilly, R. C., Busby, R. S., and Soto, R. (2003). Three forms of binding and their neural substrates: Alternatives to temporal synchrony. The unity of consciousness: Binding, integration, and dissociation, pages 168-192.

Palmer, J., Boston, B., and Moore, C. M. (2015). Limited capacity for memory tasks with multiple features within a single object. Attention, Perception, \& Psychophysics, 77(5):14881499.

Park, Y. E., Sy, J. L., Hong, S. W., and Tong, F. (2017). Reprioritization of features of multidimensional objects stored in visual working memory. Psychological science, 28(12):17731785.

Parra, M. (2017). A commentary on liang et al.'s paper with regard to emerging views of memory assessment in alzheimer's disease. Cortex, 88:198-200.

Parra, M. A., Abrahams, S., Fabi, K., Logie, R., Luzzi, S., and Sala, S. D. (2009a). Short-term memory binding deficits in alzheimer's disease. Brain, 132(4):1057-1066.

Parra, M. A., Abrahams, S., Logie, R. H., and Della Sala, S. (2009b). Age and binding withindimension features in visual short-term memory. Neuroscience Letters, 449(1):1-5.

Parra, M. A., Abrahams, S., Logie, R. H., and Della Sala, S. (2010a). Visual short-term memory binding in alzheimers disease and depression. Journal of neurology, 257(7):1160-1169.

Parra, M. A., Abrahams, S., Logie, R. H., Méndez, L. G., Lopera, F., and Della Sala, S. (2010b). Visual short-term memory binding deficits in familial alzheimers disease. Brain, 133(9):2702-2713.

Parra, M. A., Cubelli, R., and Della Sala, S. (2011a). Lack of color integration in visual shortterm memory binding. Memory \& cognition, 39(7):1187-1197.

Parra, M. A., Della Sala, S., Abrahams, S., Logie, R. H., Méndez, L. G., and Lopera, F. (2011b). Specific deficit of colour-colour short-term memory binding in sporadic and familial alzheimer's disease. Neuropsychologia, 49(7):1943-1952.

Parra, M. A., Della Sala, S., Logie, R. H., and Morcom, A. M. (2014). Neural correlates of shape-color binding in visual working memory. Neuropsychologia, 52:27-36. 
Parra, M. A., Fabi, K., Luzzi, S., Cubelli, R., Hernandez Valdez, M., and Della Sala, S. (2015a). Relational and conjunctive binding functions dissociate in short-term memory. Neurocase, 21(1):56-66.

Parra, M. A., Saarimäki, H., Bastin, M. E., Londoño, A. C., Pettit, L., Lopera, F., Della Sala, S., and Abrahams, S. (2015b). Memory binding and white matter integrity in familial alzheimers disease. Brain, 138(5):1355-1369.

Peich, M.-C., Husain, M., and Bays, P. M. (2013). Age-related decline of precision and binding in visual working memory. Psychology and aging, 28(3):729.

Pertzov, Y., Dong, M. Y., Peich, M.-C., and Husain, M. (2012). Forgetting what was where: the fragility of object-location binding. PLoS One, 7(10):e48214.

Pertzov, Y., Heider, M., Liang, Y., and Husain, M. (2015). Effects of healthy ageing on precision and binding of object location in visual short term memory. Psychology and aging, 30(1):26.

Pertzov, Y. and Husain, M. (2014). The privileged role of location in visual working memory. Attention, Perception, \& Psychophysics, 76(7):1914-1924.

Pertzov, Y., Miller, T. D., Gorgoraptis, N., Caine, D., Schott, J. M., Butler, C., and Husain, M. (2013). Binding deficits in memory following medial temporal lobe damage in patients with voltage-gated potassium channel complex antibody-associated limbic encephalitis. Brain, 136(8):2474-2485.

Piekema, C., Kessels, R. P., Mars, R. B., Petersson, K. M., and Fernández, G. (2006). The right hippocampus participates in short-term memory maintenance of object-location associations. Neuroimage, 33(1):374-382.

Piekema, C., Rijpkema, M., Fernández, G., and Kessels, R. P. (2010). Dissociating the neural correlates of intra-item and inter-item working-memory binding. PloS one, 5(4):e10214.

Pietto, M., Parra, M. A., Trujillo, N., Flores, F., Garcia, A. M., Bustin, J., Richly, P., Manes, F., Lopera, F., Ibáñez, A., et al. (2016). Behavioral and electrophysiological correlates of memory binding deficits in patients at different risk levels for alzheimers disease. Journal of Alzheimer's Disease, 53(4):1325-1340.

Quinlan, P. T. and Cohen, D. J. (2011). Object-based representations govern both the storage of information in visual short-term memory and the retrieval of information from it. Psychonomic Bulletin \& Review, 18(2):316-323.

Raffone, A. and Wolters, G. (2001). A cortical mechanism for binding in visual working memory. Journal of Cognitive Neuroscience, 13(6):766-785.

Rajsic, J., Swan, G., Wilson, D. E., and Pratt, J. (2017). Accessibility limits recall from visual working memory. Journal of Experimental Psychology: Learning, Memory, and Cognition, 43(9):1415. 
Rajsic, J. and Wilson, D. E. (2014). Asymmetrical access to color and location in visual working memory. Attention, Perception, \& Psychophysics, 76(7):1902-1913.

Rao, S. C., Rainer, G., and Miller, E. K. (1997). Integration of what and where in the primate prefrontal cortex. Science, 276(5313):821-824.

Read, C. A., Rogers, J. M., and Wilson, P. H. (2016). Working memory binding of visual object features in older adults. Aging, Neuropsychology, and Cognition, 23(3):263-281.

Rerko, L., Oberauer, K., and Lin, H.-Y. (2014). Spatial transposition gradients in visual working memory. The Quarterly Journal of Experimental Psychology, 67(1):3-15.

Rhodes, S., Parra, M. A., Cowan, N., and Logie, R. H. (2017). Healthy aging and visual working memory: The effect of mixing feature and conjunction changes. Psychology and aging, 32(4):354.

Rhodes, S., Parra, M. A., and Logie, R. H. (2016). Ageing and feature binding in visual working memory: The role of presentation time. The Quarterly Journal of Experimental Psychology, 69(4):654-668.

Riggs, K. J., Simpson, A., and Potts, T. (2011). The development of visual short-term memory for multifeature items during middle childhood. Journal of Experimental Child Psychology, 108(4):802-809.

Saiki, J. (2003a). Feature binding in object-file representations of multiple moving items. Journal of Vision, 3(1):2-2.

Saiki, J. (2003b). Spatiotemporal characteristics of dynamic feature binding in visual working memory. Vision Research, 43(20):2107-2123.

Saiki, J. (2016). Location-unbound color-shape binding representations in visual working memory. Psychological science, 27(2):178-190.

Saiki, J. and Miyatsuji, H. (2007). Feature binding in visual working memory evaluated by type identification paradigm. Cognition, 102(1):49-83.

Sala, J. B. and Courtney, S. M. (2007). Binding of what and where during working memory maintenance. Cortex, 43(1):5-21.

Sala, J. B. and Courtney, S. M. (2009). Flexible working memory representation of the relationship between an object and its location as revealed by interactions with attention. Attention, Perception, \& Psychophysics, 71(7):1525-1533.

Schneegans, S. and Bays, P. M. (2016). No fixed item limit in visuospatial working memory. cortex, 83:181-193.

Schneegans, S. and Bays, P. M. (2017). Neural architecture for feature binding in visual working memory. Journal of Neuroscience, 37(14):3913-3925. 
Schneegans, S., Spencer, J., and Schöner, G. (2016). Integrating "what" and "where": Visual working memory for objects in a scene. In Schöner, G. and Spencer, J., editors, Dynamic Thinking: A Primer on Dynamic Field Theory. Oxford University Press, New York.

Shadlen, M. N. and Movshon, J. A. (1999). Synchrony unbound: a critical evaluation of the temporal binding hypothesis. Neuron, 24(1):67-77.

Shafer-Skelton, A., Kupitz, C. N., and Golomb, J. D. (2017). Object-location binding across a saccade: A retinotopic spatial congruency bias. Attention, Perception, \& Psychophysics, 79(3):765-781.

Shafritz, K. M., Gore, J. C., and Marois, R. (2002). The role of the parietal cortex in visual feature binding. Proceedings of the National Academy of Sciences, 99(16):10917-10922.

Shen, M., Huang, X., and Gao, Z. (2015). Object-based attention underlies the rehearsal of feature binding in visual working memory. Journal of Experimental Psychology: Human Perception and Performance, 41(2):479.

Shen, M., Tang, N., Wu, F., Shui, R., and Gao, Z. (2013). Robust object-based encoding in visual working memory. Journal of vision, 13(2):1-1.

Shin, H. and Ma, W. J. (2016). Crowdsourced single-trial probes of visual working memory for irrelevant features. Journal of vision, 16(5):10-10.

Shin, H. and Ma, W. J. (2017). Visual short-term memory for oriented, colored objects. Journal of vision, 17(9):12-12.

Simmering, V. R. and Wood, C. M. (2017). The development of real-time stability supports visual working memory performance: Young childrens feature binding can be improved through perceptual structure. Developmental psychology, 53(8):1474.

Souza, A. S., Rerko, L., Lin, H.-Y., and Oberauer, K. (2014). Focused attention improves working memory: implications for flexible-resource and discrete-capacity models. Attention, Perception, \& Psychophysics, 76(7):2080-2102.

Stefurak, D. L. and Boynton, R. M. (1986). Independence of memory for categorically different colors and shapes. Perception \& Psychophysics, 39(3):164-174.

Stokes, M. G. (2015). activity-silentworking memory in prefrontal cortex: a dynamic coding framework. Trends in cognitive sciences, 19(7):394-405.

Swan, G., Collins, J., and Wyble, B. (2016). Memory for a single object has differently variable precisions for relevant and irrelevant features. Journal of vision, 16(3):32-32.

Swan, G. and Wyble, B. (2014). The binding pool: A model of shared neural resources for distinct items in visual working memory. Attention, Perception, \& Psychophysics, 76(7):21362157.

Taylor, R., Thomson, H., Sutton, D., and Donkin, C. (2017). Does working memory have a single capacity limit? Journal of Memory and Language, 93:67-81. 
Theeuwes, J., Kramer, A. F., and Irwin, D. E. (2011). Attention on our mind: The role of spatial attention in visual working memory. Acta Psychologica, 137(2):248-251.

Treisman, A. (1988). Features and objects: The fourteenth bartlett memorial lecture. The quarterly journal of experimental psychology, 40(2):201-237.

Treisman, A. (1996). The binding problem. Current Opinion in Neurobiology, 6(2):171-178.

Treisman, A., Sykes, M., and Gelade, G. (1977). Selective attention and stimulus integration. Attention and performance $\mathrm{VI}, 333$.

Treisman, A. and Zhang, W. (2006). Location and binding in visual working memory. Memory \& Cognition, 34(8):1704-1719.

Treisman, A. M. and Gelade, G. (1980). A feature-integration theory of attention. Cognitive psychology, 12(1):97-136.

Udale, R., Farrell, S., and Kent, C. (2017). No evidence for binding of items to task-irrelevant backgrounds in visual working memory. Memory \& cognition, 45(7):1144-1159.

Ueno, T., Allen, R. J., Baddeley, A. D., Hitch, G. J., and Saito, S. (2011). Disruption of visual feature binding in working memory. Memory \& Cognition, 39(1):12-23.

Vergauwe, E. and Cowan, N. (2015). Working memory units are all in your head: Factors that influence whether features or objects are the favored units. Journal of Experimental Psychology: Learning, Memory, and Cognition, 41(5):1404.

Vergauwe, E., Langerock, N., and Barrouillet, P. (2014). Maintaining information in visual working memory: Memory for bindings and memory for features are equally disrupted by increased attentional demands. Canadian Journal of Experimental Psychology/Revue canadienne de psychologie expérimentale, 68(3):158.

Vidal, J. R., Gauchou, H. L., Tallon-Baudry, C., and Oregan, J. K. (2005). Relational information in visual short-term memory: The structural gist. Journal of Vision, 5(3):8-8.

Vogel, E. K., Woodman, G. F., and Luck, S. J. (2001). Storage of features, conjunctions, and objects in visual working memory. Journal of Experimental Psychology: Human Perception and Performance, 27(1):92.

Vul, E. and Rich, A. N. (2010). Independent sampling of features enables conscious perception of bound objects. Psychological Science, 21(8):1168-1175.

Wang, B., Cao, X., Theeuwes, J., Olivers, C. N., and Wang, Z. (2016). Location-based effects underlie feature conjunction benefits in visual working memory. Journal of Vision, 16(11):1212.

Wang, B., Cao, X., Theeuwes, J., Olivers, C. N., and Wang, Z. (2017). Separate capacities for storing different features in visual working memory. Journal of Experimental Psychology: Learning, Memory, and Cognition, 43(2):226. 
Wang, X.-J. (2001). Synaptic reverberation underlying mnemonic persistent activity. Trends in neurosciences, 24(8):455-463.

Wheeler, M. E. and Treisman, A. (2002). Binding in short-term visual memory. Journal of Experimental Psychology: General, 131(1):48.

Wilson, K. E., Adamo, M., Barense, M. D., and Ferber, S. (2012). To bind or not to bind: Addressing the question of object representation in visual short-term memory. Journal of Vision, 12(8):14-14.

Wolfe, J. M. (1998). Visual search. Attention, 1:13-73.

Woodman, G. F. and Vogel, E. K. (2008). Selective storage and maintenance of an objects features in visual working memory. Psychonomic bulletin \& review, 15(1):223-229.

Woodman, G. F., Vogel, E. K., and Luck, S. J. (2012). Flexibility in visual working memory: Accurate change detection in the face of irrelevant variations in position. Visual Cognition, 20(1):1-28.

Wyble, B., Bowman, H., and Nieuwenstein, M. (2009). The attentional blink provides episodic distinctiveness: sparing at a cost. Journal of Experimental Psychology: Human Perception and Performance, 35(3):787.

Wyble, B., Potter, M. C., Bowman, H., and Nieuwenstein, M. (2011). Attentional episodes in visual perception. Journal of Experimental Psychology: General, 140(3):488.

$\mathrm{Xu}$, Y. (2002a). Encoding color and shape from different parts of an object in visual short-term memory. Perception \& psychophysics, 64(8):1260-1280.

$\mathrm{Xu}, \mathrm{Y}$. (2002b). Limitations of object-based feature encoding in visual short-term memory. Journal of Experimental Psychology: Human Perception and Performance, 28(2):458.

$\mathrm{Xu}, \mathrm{Y}$. (2006). Understanding the object benefit in visual short-term memory: The roles of feature proximity and connectedness. Perception \& Psychophysics, 68(5):815-828.

Xu, Y. and Chun, M. M. (2006). Dissociable neural mechanisms supporting visual short-term memory for objects. Nature, 440(7080):91.

Yeh, Y.-Y., Yang, C.-T., and Chiu, Y.-C. (2005). Binding or prioritization: The role of selective attention in visual short-term memory. Visual Cognition, 12(5):759-799.

Zokaei, N., Heider, M., and Husain, M. (2014). Attention is required for maintenance of feature binding in visual working memory. Quarterly journal of experimental psychology, 67(6):1191-1213. 